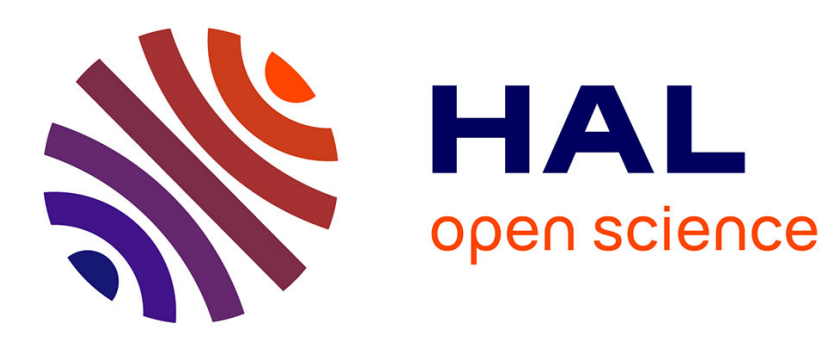

\title{
Aux origines du bouddhisme siamois
}

Michel Lorrillard

\section{To cite this version:}

Michel Lorrillard. Aux origines du bouddhisme siamois. Bulletin de l'Ecole française d'ExtrêmeOrient, 2000, 87 (1), pp.23-55. 10.3406/befeo.2000.3469 . hal-02358498

\section{HAL Id: hal-02358498 https://hal.science/hal-02358498}

Submitted on 12 Nov 2019

HAL is a multi-disciplinary open access archive for the deposit and dissemination of scientific research documents, whether they are published or not. The documents may come from teaching and research institutions in France or abroad, or from public or private research centers.
L'archive ouverte pluridisciplinaire HAL, est destinée au dépôt et à la diffusion de documents scientifiques de niveau recherche, publiés ou non, émanant des établissements d'enseignement et de recherche français ou étrangers, des laboratoires publics ou privés. 


\section{Aux origines du bouddhisme siamois [Le cas des buddhapāda]}

In: Bulletin de l'Ecole française d'Extrême-Orient. Tome 87 №1, 2000. pp. 23-55.

Citer ce document / Cite this document :

Lorrillard Michel. Aux origines du bouddhisme siamois [Le cas des buddhapāda]. In: Bulletin de l'Ecole française d'ExtrêmeOrient. Tome 87 N¹, 2000. pp. 23-55.

doi : $10.3406 /$ befeo.2000.3469

http://www.persee.fr/web/revues/home/prescript/article/befeo_0336-1519_2000_num_87_1_3469 
Résumé

Michel Lorrillard

Aux origines du bouddhisme siamois

Le cas des buddhapâda

Le bouddhisme siamois ancien - en particulier celui qui s'est développé dans la région de Sukhothai se distingue par un culte important voué aux empreintes du Buddha (buddhapāda). L'étude des premières d'entre elles, et des inscriptions qui leur sont liées, montre très clairement une influence littéraire cinghalaise. Cette influence a cependant varié dans le temps. D'abord relayée par la Birmanie, elle a été marquée par des textes pāli de composition tardive et a été étroitement associée à une tradition artistique née à Pagan.

Par la suite, une iconographie nouvelle et indépendante se développa, inspirée en grande partie par les œuvres canoniques et les commentaires. Certaines références se révèlent toutefois plus difficiles à identifier et relèvent apparemment de traditions locales, fort étrangères à l'orthodoxie cinghalaise. Le buddhapāda peut alors devenir le support de conceptions " tantriques » et se rattacher à un fonds de pratiques reconnues aujourd'hui comme très anciennes. Dans tous les cas, les témoignages relatifs aux empreintes du Buddha nous offrent de précieuses informations pour évaluer la culture religieuse siamoise la plus ancienne.

\section{Abstract}

Michel Lorrillard

Towards the origins of Siamese Buddhism

The case of the buddhapāda

A distinguishing trait of ancient Siamese Buddhism - and more precisely the form of Buddhism which developed in Sukhothai - is the important cult of the Buddha's footprints \{buddhapāda). The study of the first footprints and related inscriptions shows a very clear Singhalese literary influence. This influence changed with time. Coming first through Burma it was connected with late Pali works and related to an artistic tradition that originated in Pagan. Later, a new and independant iconography developed, inspired mainly by canonical scriptures and commentaries. Some of the references, however, are not easily identified and may come from local traditions, alien to Singhalese orthodoxy. The buddhapāda may then have become a basis for "tantric" conceptions and been linked to a set of practices now acknowledged to be ancient in origin. Whatever was the case, the Buddha's footprints offer precious information about Siamese religious culture from earliest times. 


\title{
Aux origines du bouddhisme siamois \\ Le cas des buddhapāda
}

\author{
Michel LORRILLARD*
}

Les témoignages les plus anciens concernant le bouddhisme siamois - en particulier ceux de la région de Sukhothai - mettent en évidence un culte important voué aux empreintes du Buddha (buddhapäda) ${ }^{\prime}$. Présenté au milieu du XIV siècle comme un fait nouveau, ce culte bénéficia pourtant d'une légitimité religieuse immédiate et incontestée. Il est très certainement à mettre en rapport avec des traditions cinghalaises qui, via la Birmanie, revivifièrent à partir du XII ${ }^{\mathrm{c}}$ siècle le bouddhisme theravāda d'Asie du Sud-Est ${ }^{2}$. De la même façon qu'aux inscriptions qui évoquent le sujet, il est nécessaire d'accorder aux premières empreintes «siamoises » du Buddha - dont plusieurs ont été conservées une véritable attention. Elles nous renseignent en effet sur des croyances et des pratiques que les siècles ont très largement modifiées. Elles reflètent surtout un patrimoine intellectuel ancien, et éclairent du même coup des pans de l'histoire religieuse du peuple thaï.

\section{Les références épigraphiques}

Nous n'avons aucune donnée écrite relative aux empreintes du Buddha en milieu thaï qui soit antérieure à l'inscription de Nagara Jum, rédigée le 23 juin $1357^{3}$. Celle-ci fait état à cette date de la présence dans la région de Sukhothai de quatre pādalakșana ${ }^{4}$, tous

* Je voudrais remercier MM. B. Buddharaksa, S. Collins, O. von Hinüber et P. Skilling d'avoir attiré mon attention sur un certain nombre de points.

1. Ne seront considérées ici que les empreintes antérieures (ou reconnues comme telles) au XVI siècle. Ces objets sacrés se sont en effet multipliés à partir de la période d'Ayuthya. Une liste non exhaustive en recense aujourd'hui cent cinquante-cinq en Thaillande (cf. Phutthabat Lakkhana Lè Roy Phra Phutthabat Nai Prathet Thai, Bangkok, 2536 (1993), p. 45-49).

2. Sur la question particulière de la cinghalisation du bouddhisme thaï, on se référera à $G$. Coedès, Les États hindouisés d'Indochine et d'Indonésie, Paris, 1964; A. B. Griswold et P. na Nagara, Epigraphic and Historical Studies, [recueil des articles parus sous le même titre dans le Journal of the Siam Society], Bangkok, 1992 ; F. Bizot, Les traditions de la pabbajjā en Asie du Sud-Est, Göttingen, 1988.

3. Des études importantes ont été consacrées à cette inscription: G. Cœdès, "L'inscription de Nagara Jum », Journal of the Siam Society (JSS), 13/3, 1919, p. 1-43 ; G. Cœdès, Recueil des inscriptions dı Siam I, Bangkok, 1924, p. 77-90 ; A. B. Griswold \& P. na Nagara, « Epigraphic and Historical Studies No. 11 Part I : The Epigraphy of Mahādharmarājā I of Sukhodaya », JSS, 61/1, 1973, p. 79-111 ; Charük Samay Sukhothai, 2527 E.B., p. 26-39. Pour la date, on se référera également à J. C. Eade, The Thai Historical Record, A Computer Analysis, Tôkyô, 1996, p. 5-6.

4. Litt. : «marques (ou signes) du pied». Dans le contexte qui nous occupe, le composé désigne plus précisément une empreinte du Buddha avec ses 108 marques auspicieuses. Si l'appellation pādalakșana est sanskrite (équivalent pāli : pādalakkhana), il est intéressant de remarquer que la tradition relative aux 108 marques semble quant à elle être exclusivement pāli. Le composé sanskrit/pāli buddhapāda («pied du 
situés sur des sommets. Cette position élevée s'explique par le fait que les objets concernés sont mis explicitement en rapport avec la fameuse empreinte du Sumanakūta ${ }^{5}$ à Ceylan qui est dite leur avoir servi directement de modèle ${ }^{6}$. De fait, l'une des quatre collines abritant ces empreintes est justement appelée "Sūmanakūta ». Les trois autres localisations sont un sommet de Si Satchanalai (dont le nom n'a pas été conservé par la pierre), le sommet du Khau Nang Thong, à Bang Phan, et le sommet d'une colline à Pak Phra Bang. Il est précisé que des inscriptions sont placées en chacun de ces lieux. Toutes les empreintes sont dites avoir été installées par le Phrayā Dharmikarājā - souverain qu'il faudra probablement identifier, à la suite de G. Cœdès, avec Lödaiya, mort en 1347.

\section{Khau Nang Thong, Pak Phra Bang et Sumanakūtaparvata}

Des quatre pādalaksana cités dans l'inscription de Nagara Jum, trois ont été identifiés, dont deux de façon à peu près certaine ${ }^{7}$. Celui du Khau Nang Thong, près de Kampheng Phet, a été envoyé à Bangkok en 1923. Il porte quelques caractères, mais ceux-ci ont été déclarés peu lisibles par G. Cœdès ${ }^{8}$ et n'ont, semble-t-il, jamais donné lieu à une étude. Celui de la colline de Pak Phra Bang est toujours in situ. L'endroit a été identifié avec la hauteur connue aujourd'hui sous le nom de Khau Kôp, à Nakhon Sawan, à côté du confluent de la Mè Nam Ping et de la Mè Nam Nan ${ }^{9}$. Une stèle se trouvait à côté de

Buddha ») a un sens plus générique : il désigne également une empreinte du Buddha (le syntagme thaï « roy tīn phra phutthabāt » apparaît alors plus complet), mais celle-ci pourra être dénuée des 108 marques et présenter une iconographie fort différente. On notera par ailleurs l'emploi des formules « roy tin phra phuttha chao » (empreinte du pied du Buddha) et « roy fā tin phra phuttha chao » (empreinte de la plante du pied du Buddha). Pour les buddhapāda les plus anciens, on consultera avec intérêt A. M. Quagliotti, Buddhapadas. An Essay on the Representations of the Footprints of the Buddha with a Descriptive Catalogue of the Indian Specimens from the $2^{\text {md }}$ Century B.C. to the $4^{\text {th }}$ Century A.D., Kamakura, Institute of the Silk Road Studies, 1998. Un appendice est consacré à certaines pièces trouvées en Asie du SudEst.

5. Litt. : «Pic de Sumana ». L'état de la pierre ne permettant qu'une lecture partielle, le nom a été en grande partie restitué. L'endroit auquel il est fait référence est connu dans les sources occidentales sous le nom de «Pic d'Adam ".

6. Ce buddhapāda (que les hindouistes et les chrétiens prennent respectivement pour une empreinte de Śiva ou d'Adam) est en fait une cavité naturelle d'environ 1,65 m de long, sans caractéristiques particulières. A. B. Griswold \& P. ṇa Nagara (op. cit., p. 111), qui se basent sur un ouvrage du XIX siècle, rapportent qu'elle fut protégée par un couvercle (aujourd'hui disparu) sur lequel étaient gravées les 108 marques auspicieuses. Il est sans doute imprudent d'affirmer à la suite des deux historiens que c'est ce couvercle qui fut copié.

7. Le buddhalakșana du sommet de Si Satchanalai n'a pas été retrouvé, à moins qu'il ne s'agisse -comme cela a été suggéré (cf. Nangsü Thiralïk Phra Rajaphithi Yok Chula Mongkhut Lè Somphot Phra Phutthabat Changvat Saraburi, Bangkok, 1999, p. 34) ․ de l'une des deux empreintes conservées aujourd'hui au Wat Sri Khom Kham de Phayao. La relative proximité de cette cité, ainsi que la ressemblance stylistique de la pièce avec les trois autres, constituent à ce propos des arguments favorables. La décoration en est cependant plus riche : vingt panneaux, figurant des Buddha ou des divinités, entourent en effet l'empreinte proprement dite.

8. Recueil ... (op. cit.), p. 90. Selon le service d'information du musée national de Bangkok, c'est ce buddhapāda qui figure (sans référence) parmi les collections de la salle consacrée à l'art de Sukhothai. Les caractères relevés par G. Cœdès sont khmers. Ils sont répartis dans une douzaine de lignes aujourd'hui à peine visibles. L'empreinte, qui est en pierre, possède les 108 marques auspicieuses. Le dessin d'un personnage debout et en prière est également gravé.

9. À la page 90 du Recueil ... (op. cit.), G. Cœdès présente cette identification comme étant certaine (« il s'agit de la colline Khau Kop ») alors qu'à la page 145, il apparaît plus prudent (« ... une empreinte du pied du Buddha, qui semble correspondre à celle que l'inscription de Nagara Jum signale à Pāk Yam 
l'empreinte, mais il n'est pas certain que ce soit celle dont il est question dans l'inscription de Nagara Jum. Datant apparemment du règne de Ldaiya (1347-1368/74 ?), son objet, en effet, n'est pas de commémorer l'installation du buddhapāda, mais celle d'un dispositif le protégeant, de même que la construction d'un vihāra et d'un cetiya ${ }^{10}$.

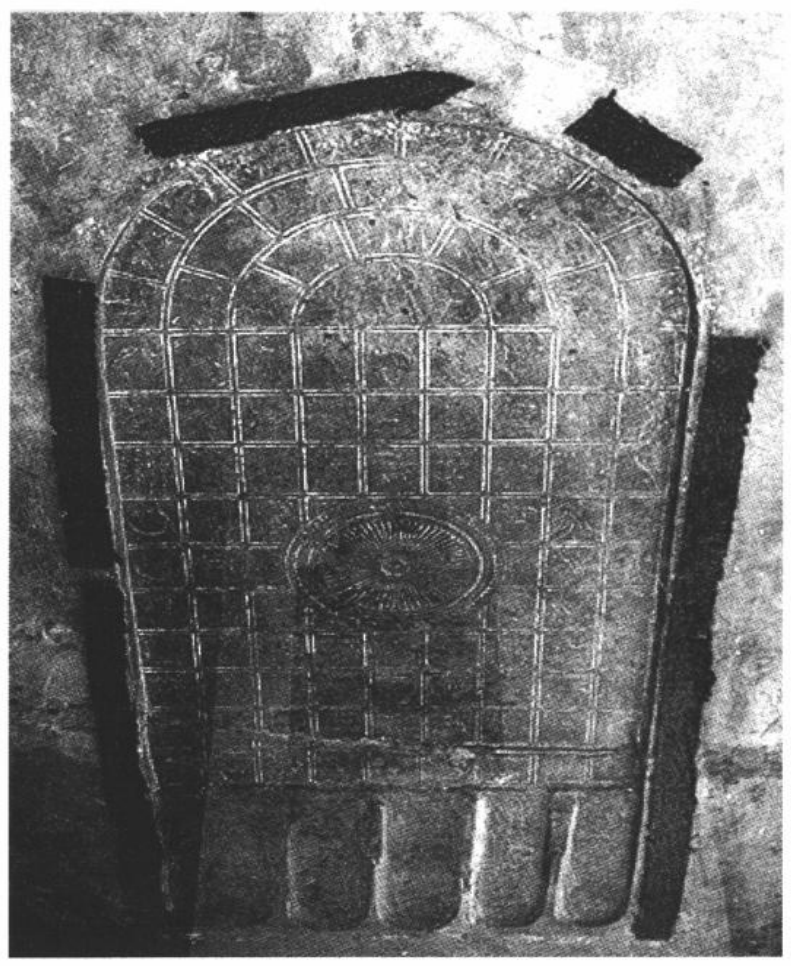

Buddhapāda de Pak Phra Bang (Nakhon Sawan)

(Fine Arts Department)

La référence à un pādalakșana sur le sommet d'une colline portant le nom de Sumanaküta a fait naître dans les études thaïes une certaine confusion qui, aujourd'hui encore, persiste. Le sujet est en effet évoqué par deux inscriptions : la stèle de Nagara Jum, dont nous avons vu qu'elle datait de 1357, et la stèle du Sumanakūtaparvata, conservée depuis 1908 à la Bibliothèque nationale de Bangkok ". Cette dernière est datée d'environ 1369. Elle relate entre autres événements l'installation (ou la réinstallation?)

Brah Păn »). Ses successeurs n'ont cependant pas mis en doute cette identification. L'empreinte est située aujourd'hui au centre du complexe que forme le Wat Voranart Banphot. Elle présente un schéma général identique à celle du Khau Nang Thong.

10. G. Coedès, Recueil ... (op. cit.), p. 145-149 ; A. B. Griswold \& P. ṇa Nagara (op. cit.), p. 112118 ; Charïk Samay Sukhothai, p. 182-185. Le texte qui nous concerne figure sur la face 1 de la stèle. Celle-ci étant très endommagée, seule une lecture restituée du composé (pādala)kṣana a permis de reconnaître une référence à l'empreinte. Il semble que cette dernière avait été abandonnée quelque temps (elle pourrait donc être antérieure au règne de L Laiya) et que l'on procéda alors à des travaux de rénovation afin de la mettre une nouvelle fois en valeur. A. B. Griswold et P. na Nagara évoquent la construction d'un mandapa. Dans un passage où les lacunes sont très importantes, il est également question de la présence d'un arbre de la bodhi au sommet de la colline «Sūmana(kūta) ». Cette référence n'a guère été commentée.

11. G. Cœdès, Rectueil ... (op. cit.), p. 123-130 ; A. B. Griswold \& P. ṇa Nagara, « Epigraphic and Historical Studies, No. 1 1, Part II : The Epigraphy of Mahādharmarājā I of Sukhodaya », JSS, 61/2, 1973, p. 100-123; Chariik Samay Sukhothai, p. 80-91. 
par le Phrayā Śrī Sūryavamśa Mahādharmarājādhirājā (Ldaiya) d'une empreinte du Buddha sur le Sumanakūtaparvata ${ }^{12}$ en 1359. Antérieurement ou postérieurement à cette date (on ne saurait en décider d'une façon sûre), il est également fait référence à la sculpture ou à la gravure de l'objet sacré. La provenance exacte de la stèle, malheureusement, n'est pas connue. On a supposé qu'elle était originaire soit du Khau Phra Bat Noy, soit du Khau Phra Bat Yai, situés respectivement à l'ouest et au sud-ouest de Sukhothai. Une empreinte était installée sur chacune de ces collines, celle de la seconde ayant été par la suite transportée au Wat Traphang Thong ${ }^{13}$. A. B. Griswold et P. na Nagara ne doutent pas que ce soit cette dernière qu'il faille identifier avec le pādalaksaña dont il est question dans la stèle du Sumanakūtaparvata ${ }^{14}$. G. Codès est beaucoup plus partagé. Avec une grande prudence, il rapporte que le nom de «Sūmanakūta » - tel qu'il apparaît dans l'inscription de Nagara Jum - désigne "peut-être la colline nommée aujourd'hui Brah Pāda Hñai ${ }^{15}$. En même temps, il trouve également vraisemblable l'hypothèse selon laquelle la stèle du Sumanakutaparvata pourrait provenir du «Khau Brah Pāda Noy (...), où l'on vénère encore une empreinte du pied du Buddha, analogue aux autres empreintes fondées par Dharmarāja ${ }^{16}$. Il est manifeste que G. Codès, au moment où il rédigeait ces lignes (c'est-à-dire peu avant 1924), voyait dans les pādalakṣana du Mont Sumanakūta auxquels il est fait référence dans les deux inscriptions un même et unique objet sacré installé par Ldaiya. Sur ce dernier point, cependant, il changea par la suite d'avis et se rangea à la thèse développée par le Phya Nakhon Phra Ram selon laquelle c'était Lödaiya, appelé également Dharmikarājā, qui était l'initiateur de l'installation des empreintes faites à l'imitation du buddhapāda de Ceylan ${ }^{17}$. Il est intéressant, à ce stade, de relever un détail qui a peut-être quelque importance. Dans sa traduction de la stèle du Sumanakūțaparvata publiée en 1924, G. Cœedès restitue une phrase où l'auteur de l'inscription dit venir rendre hommage à l'empreinte " que son père avait placée sur le sommet de cette colline ». Étant encore convaincu que l'initiateur de l'installation de l'empreinte était Ldaiya, il déduit alors que l'auteur de l'inscription était le fils de ce dernier - ce qui apparaît tout à fait étonnant dans la mesure où le personnage se donne le titre de Śrī Sūryavamiśa Dharmarājādhirāja qui désigne précisément Ĺdaiya dans d'autres inscriptions. Quelques quarante années plus tard, P. na Nagara donne cependant une lecture différente du mot que Cœdès avait restitué et traduit par «son père» et propose à la place la traduction «lui-même ${ }^{18}$. Bien qu'il ne soit pas tout à fait convaincu sur le plan paléographique, Cœedès se range tout de même à l'avis de P. ṇa Nagara et

12. Litt.: «montagne (nommée) pic de Sumana ». Nous avons restitué l'orthographe sanskrite (P. Sumanakūtapabbata). Dans l'inscription, le nom est noté « Sūmanakūțaparrbata ».

13. Cette empreinte est munie des 108 signes auspicieux. Ceux-ci sont disposés selon un schéma qui est pratiquement identique à celui de l'empreinte du Khau Nang Thong.

14. Op. cit., p. 542. Les deux auteurs n'exposent malheureusement pas leurs arguments.

15. Op. cit., p. 90.

16. Op. cit., p. 123. Dans les collections du Musée de Sukhothai figure aujourd'hui un buddhapāda en pierre présenté comme étant originaire du Wat Phra Bat Noy. Il semble que ce soit la pièce mentionnée par G. Cœdès. Si les 108 signes auspicieux sont représentés, leur disposition s'avère cependant très différente de celle que l'on observe sur les buddhapāda du Khau Nang Thong et du Wat Traphang Thong.

17. Les États hindouisés d'Indochine et d'Indonésie, 1964, p. 398. On comprendra donc que ce sont les quatre pädalakșạa de l'inscription de Nagara Jum, le quatrième étant également celui de l'inscription du Sumanakūtaparvata. Dans son article «Who was Dharmarājā I of Sukhothai ? » (JSS, 28, 1935, p. 214-220), le Phya Nakhon Phra Ram n'évoque en fait explicitement que le buddhapāda du Mont Sumanakūta de Sukhothai.

18. « $h \bar{a} k »$ à la place de «j(a)n(a)k(a)». 
reconnaît Ldaiya non seulement comme le seul souverain dont il soit question dans l'inscription, mais également comme l'initiateur du buddhapāda du Mont Sumanakūta ${ }^{19}$. Il ne semble pas qu'à ce moment-là, l'orientaliste ait également remis en question son attribution à Lödaiya des quatre buddhalakșana de l'inscription de Nagara Jum. Une dissociation s'est probablement opérée dans son esprit, et il est possible qu'il ait finalement conclu à l'existence de deux empreintes du Buddha distinctes liée chacune à un sommet portant le même nom de Sumanaküta. Il pourrait alors s'agir des deux montagnes Khau Phra Bat Noy et Khau Phra Bat Yai qui, toutes deux, ont révélé un buddhapāda ancien ${ }^{20}$.

En attribuant la paternité des empreintes de Buddha au seul Ldaiya, A. B. Griswold et P. ṇa Nagara partent sur une base de travail différente ${ }^{21}$. La principale difficulté était peutêtre de concilier des données chronologiques contradictoires. L'inscription du Sumanakūtaparvata indique en effet que le pädalaksana fut installé en 1359, alors que l'inscription de Nagara Jum mentionne déjà sa présence sur la colline en 1357. L'anachronisme, selon les deux historiens, n'est cependant qu'apparent. Ils proposent en effet de voir dans le dernier passage de l'inscription de Nagara Jum un «post-script » qu'ils datent d'environ 1360 .

La stèle du Sumanakūtaparvata, en même temps qu'elle offre un certain nombre de détails touchant à l'installation d'un buddhapāda, révèle d'une façon implicite des informations utiles pour une meilleure compréhension du contexte. Il est par exemple intéressant d'essayer de déterminer au plus près la valeur que pouvait avoir dans la conscience du rédacteur (en partie représentative de la conscience collective de l'époque) l'empreinte à laquelle il est fait référence. La première phrase de l'inscription apparaît à ce sujet significative. Plutôt que de mettre l'accent sur l'objet sacré, elle commence en effet par donner le nom de la colline - celui-ci n'ayant pas été créé de toute pièce (son importance aurait alors été moindre), mais ayant été emprunté à un lieu que la tradition a rendu célèbre : le Pic Sumanakūta à Ceylan ${ }^{22}$. Il n'y a dans cette pratique de copier un

19. Lettre du 19 septembre 1964 adressée par G. Cœdès à P. na Nagara (incluse dans le texte de la communication de P. na Nagara à l'occasion du colloque «George Coedès aujourd'hui », Bangkok, 9-10 septembre 1999).

20. On peut remarquer que le revirement de Cœdès ne donna jamais lieu à une rectification publiée, intervenant sans doute trop tard pour qu'une note soit insérée dans la dernière édition (1964) des États hindouisés. Nous pensons pour notre part que la première lecture de l'orientaliste - celle qui fait mention du "père », initiateur de l'installation du buddhapāda - garde une certaine pertinence. Le père serait alors Lödaiya et non pas Ldaiya, comme Coedès l'a d'abord cru (en 1924). L'identification de Ldaiya comme étant l'auteur de l'inscription serait alors prouvée non pas par la traduction "lui-même " (proposée par P. ṇa Nagara) mais par le titre de Śrī Sūryavaṃśa Dharmarājādhirājā que se donne l'auteur (cet argument a déjà été avancé par le Phya Nakhon Phra Ram, op. cit., p. 218). Il y aurait ainsi conservation d'une logique vis-à-vis de l'inscription de Nagara Jum. Le seul problème tient à la date de 1359 présentée comme étant celle de l'installation. L'inscription du Sumanakūtaparvata est cependant lacunaire à cet endroit et il pourrait s'agir en fait d'une réinstallation.

21. Les deux historiens identifient le Phrayā Dharmikarājā de l'inscription de Nagara Juṃ avec Mahādharmarājā I, c'est-à-dire Ĺdaiya, contrairement à G. Codès qui y voit Lödaiya.

22. C'est sous cette forme que le nom apparaît en premier dans le Mahāvamsa. Comme le signale cependant W. Geiger (Culture of Ceylon in Medieval Times, p. 2), «between the eleventh and thirteenth centuries the name Sumanaküta alternates in the chronicle with Samantaküta, perhaps because the peak is visible from all sides (samantato) ". Cette remarque est importante car dans l'inscription du buddhapāda du Wat Pavaranivesa, datée de 1426, c'est le nom Samantaküța qui est employé à la place de Sumanakūta (cf. infra). L'endroit est également connu sous les noms de Sumanagiri, Samantagiri et Sumanācala (cf. G. P. Malalasekera, Dictionary of Pāli Proper Names, vol. II, p. 1044-1045). 
toponyme appartenant au monde indien rien qui doive nous surprendre. Elle existait déjà aux premiers temps de l'indianisation et subsiste encore aujourd'hui. Ce qui apparaît en fait original dans le document qui nous concerne, c'est que son auteur explicite l'emprunt du nom en opérant un lien direct entre la colline de Sukhothai et le pic cinghalais. En quoi consiste ce lien? Il tient dans le fait qu'on serait allé copier l'empreinte du pied avec lequel le Buddha aurait foulé le sommet du Sumanakūta et que l'on aurait installé la copie sur la colline siamoise. Il semble que personne ne se soit jusqu'ici véritablement prononcé sur l'authenticité de l'information contenue dans ce passage. A. B. Griswold et P. na Nagara paraissent cependant l'accepter tacitement, d'autant plus qu'ils forcent quelque peu la traduction lorsqu'ils évoquent un émissaire qui serait allé faire des impressions à Lankā. Les deux historiens ne semblent pas avoir été gênés par la totale absence de détails entourant cette affaire. Il ne fait pourtant aucun doute qu'une entreprise aussi importante que l'envoi d'une mission siamoise à Ceylan aurait suscité de la part du rédacteur un peu plus de commentaires. On peut objecter qu'elle avait peut-être déjà eu lieu depuis un certain temps, qu'elle avait pu être célébrée ailleurs et qu'elle était alors connue de tous les habitants de l'époque. Dans la partie finale de l'inscription de Nagara Jum, il est en effet écrit que Phrayā Dharmikarāja (Lödaiya ?) lui-même avait fait imprimer l'empreinte que le Buddha laissa à Sinhāla sur le sommet du Sumanakūta. C'est cette copie qui avait alors permis de réaliser les quatre empreintes dispersées sur le territoire de Sukhothai. La même inscription (cette fois dans ses premières lignes) évoque une importante relique (dhätu) présentée comme authentique et qui serait également venue de Lankā en même temps qu'une pousse de l'arbre de la bodhi. La rhétorique est cependant la même et ne présente pas de détails autres que ceux que la tradition a légués. En fait, il faut se garder de prendre à la lettre nombre de passages stéréotypés contenus dans les premières inscriptions de Sukhothai. Ceux-ci procèdent à l'évidence de modèles littéraires qu'il est par ailleurs essentiel d'identifier si l'on veut avoir une meilleure approche des traditions bouddhiques répandues originellement en milieu thaï. La référence au Pic Sumanakūta fait partie de ces traditions. Elle est issue de la littérature pāli du courant mahāvihāra et figure dans les premières pages du Mahāvamsa ${ }^{23}$, la célèbre chronique cinghalaise connue en Birmanie depuis au moins le $X_{I I}{ }^{e}$ siècle ${ }^{24}$. Un certain nombre d'indices permettent de dire que le même texte servait déjà de source d'inspiration à Sukhothai dans la première moitié du $X V^{e}$ siècle et peut-être même à la fin du XIII ${ }^{25}$. La filiation opérée par les scribes de

23. The Mahāvansa or the Great Chronicle of Ceylon, (trad. W. Geiger), Pāli Text Society, 1912, p. 8 (ch. I : 77-78).

24. Des illustrations inspirées par le Mahāvamsa figurent dans le temple de Myinkaba Kubyauk-gyi, que l'on date des environs de 1113 A. D. Cf. G. H. Luce, Old Burma. Early Pagan, vol. I, p. 62, 105, $246,377,379-382$.

25. L'inscription du Wat Sri Chum, que l'on date avec une grande vraisemblance du règne de Lödaya, contient un certain nombre de références à des sites bouddhiques qui semblent issues directement du Mahāvanısa. Nous ne suivons pas A. B. Griswold et P. na Nagara (et après eux quelques auteurs) qui prennent le texte de cette inscription à la lettre et pensent que son auteur serait allé à Ceylan et en Inde pour y visiter des lieux sacrés et procéder à des restaurations. L'inscription de Ram Khamhacng (tenue pour authentique) est inspirée elle aussi par des traditions littéraires. Il est possible que la référence à une cloche que l'on sonnait pour appeler la justice du roi procède déjà du Mahāvamısa (Cf. Piriya Krairiksh, "A Reassessment of the Inscription of King Ram Khamhaeng », in The Ram Khamhaeng Controversy, p. 75).

La connaissance par les Thaïs à une date ancienne du Mahāvamsa est beaucoup plus évidente que celle de la Samantakütavannanā, composée à Ceylan à la fin du XIII" siècle ou au début du XIVe. Cet ouvrage poétique décrit la visite du Buddha à Ceylan, et en particulier le moment où il laissa son empreinte sur le Mont Samantaküta. Cf. O. von Hinüber, A Handbook of Päli Literature, 1996, p. 97, § 
Sukhothai avec Ceylan était alors purement conventionnelle, mais en même temps obligatoire, car légitimante. Comment en effet appeler les fidèles à honorer une relique ou une empreinte si celle-ci n'était pas reliée directement au Buddha lui-même ${ }^{26}$ ? On ne pourra pour autant parler franchement de falsification. L'iconographie des premières empreintes du Buddha de Sukhothai, si elle ne put être issue directement de Ceylan - ce que confirme les données de l'archéologie et de l'histoire de l'art ${ }^{27}$-, pouvait reposer sur d'autres modèles qu'une tradition locale reconnaissait également comme originaire de l'île. On a parlé à ce propos d'une influence possible de Pagan ${ }^{28}$. C'est l'hypothèse qui, en effet, paraît aujourd'hui la plus plausible. Dans l'état actuel des recherches, le territoire thaïlandais n'offre en tout cas aucun exemple de buddhapāda susceptible d'avoir servi de prototype aux premières empreintes de Sukhothai ${ }^{29}$.

Si la référence aux marques caractéristiques d'une empreinte du Buddha était déjà contenue dans le terme même de pādalakșana, c'est dans la stèle du Sumanakūtaparvata que l'on explicite pour la première fois leur nombre. Il y est en effet question des 108 signes ou dessins (« lāy ») destinés à être honorés des dieux (devatā) et des hommes (le terme est effacé mais peut être restitué de façon pratiquement certaine), et dont on nous précise qu'ils sont de couleur éclatante («sī san ») ${ }^{30}$. La tradition relative aux 108 marques

198 ; et K.R. Norman, Pāli Literature, 1983, p. 159. La Samantakütavaṇnanā a été éditée par C. E. Godakumbura (London, 1958) et traduite en anglais par A. A. Hazlewood (In Praise of Mount Samanta, London, 1986).

26. Par la suite, les buddhapāda installés en pays thaï ne trouveront plus leur légitimité dans le fait qu'ils soient (prétendus être) des copies de l'empreinte du Sumanakūta, mais dans le fait qu'ils auront été laissés par le Buddha lui-même lors d'une visite. Des chroniques, rédigées dans une intention légitimante, relatent alors le fait d'une façon plus ou moins détaillée. C'est par exemple le cas pour le célèbre buddhapāda de Saraburi.

27. J. Boisselier (La sculpture en Thaïlande, p. 137) rapporte que les empreintes et les couvreempreintes de bronze exécutés à Sukhothai aux XIV et XVe siècles ne révèlent pas l'influence de l'art cinghalais.

28. Ibid. Si l'on compare les buddhapāda du Wat Traphang Thong, du Khau Kôp et du Khau Nang Thong avec ceux des temples Lokananda Zedi, Shwézigon, Lokahteikpan et Theimazi, à Pagan ( $c f$. Luce, $o p$. cit., vol. III, pl. 92 b et $93 \mathrm{a}, \mathrm{b}, \mathrm{c}$ ), on constate effectivement des ressemblances stylistiques flagrantes (en particulier dans la disposition des 108 signes auspicieux) que l'on ne retrouvera plus dans les empreintes siamoises postérieures.

29. Quelques rares empreintes datant de la période de Dvāravatī ont été retrouvées mais ont peu à voir avec les buddhapāda de Sukhothai. Celle qui semble la plus ancienne (VII-VIII siècles) est située à Sra Morakot, dans la province de Prachinburi, et se présente en fait comme une double empreinte avec une roue gravée à la place des deux centres. Cette caractéristique peut être également brahmanique, mais le site a révélé d'autres vestiges qui attestent une culture bouddhique. Quatre autres empreintes, avec une fleur de lotus gravéc en leur centre, proviennent de la province d'Udon Thani où une culture mône était bien représentée. Assez récemment, on a découvert par ailleurs une empreinte à Khao Taphao, dans la province de Lopburi, que l'on date (selon des critères apparemment fragiles) du XII au XIV' siècle. Il s'agit manifestement d'une cavité rocheuse que l'on a retouchée en creusant distinctement les contours de cinq orteils. L'empreinte est totalement dépourvue de signes, même de la roue. En ce qui concerne le Cambodge, il semble bien que les buddhapāda ne soient apparus qu'à partir de la période postangkorienne. Quclques empreintes antérieures relèvent de l'art brahmanique préangkorien (Cf. F. Bizot, «La figuration des pieds du Buddha au Cambodge", Études Asiatiques, 25, 1971, p. 407-439). M. Giteau (Iconographie du Cambodge post-angkorien, EFEO, 1975, p. 45) signale «que les figures sculptées sur les Buddhapäda (du Cambodge), et plus particulièrement sur les images d'Angkor Vat, doivent peu à l'esthétique khmère, mais sont étroitement apparentées à l'art d'Ayuthyā ".

30. On comprendra que le buddhapāda était soit polychrome, soit recouvert d'une peinture particulièrement voyante. Than Tun («The Buddha's Footprints in Burma », Journal of Asian and 
semble appartenir au bouddhisme d'expression pāli ${ }^{31}$, même si elle ne figure pas dans les livres du Tipitaka ou de ses premiers commentaires. Selon l'archéologue birman U Mya, elle apparaît dans deux textes relativement tardifs : un commentaire du Jinālañkāra (Jināl-t ) et un commentaire de l'Anāgatavamsa ${ }^{32}$. Le premier semble avoir été composé en 1156 par un auteur né à Ceylan ${ }^{33}$. Au vu de ce que nous connaissons des relations entre l'île et la Birmanie à cette époque, il n'est pas invraisemblable qu'il soit parvenu dans cette dernière très vite. Il est fait référence à ce texte dans la Jinakālamālī rédigée au Lanna en $1516^{34}$. Il existe plusieurs commentaires de l'Anāgatavamsa $(A n \bar{a} g-a)^{35}$. Celui dont il est question semble être la Samantabhaddikā, également référencé sous le nom de Samantabhaddatīk $\bar{a}^{36}$. Il est possible qu'il ait été écrit par Upatissa de Ceylan, l'auteur probable du Mahăbodhivamsa, aux alentours du $\mathrm{X}^{\mathrm{e}}$ siècle $^{37}$. Il est extrêmement intéressant de constater que la Traibhümikathā, traité cosmologique siamois attribué à Ldaiya, cite parmi les trente-trois textes présentés comme étant ses sources d'inspiration le Jinālañkāra, l'Anāgatavamsa et le Bodhivamsa ${ }^{38}$. Il est donc tout à fait vraisemblable que les commentaires des deux premiers aient été connus à Sukhothai au XIV ${ }^{\mathrm{e}}$ siècle.

La stèle du Sumanakūtaparvata indique que quiconque ira saluer avec un cœur rempli de foi l'empreinte de la plante du pied du Buddha au sommet de la colline de Sukhothai parviendra de façon certaine aux « trois accomplissements » (« sampatti dañ sām »). Ceuxci sont apparemment détaillés dans le passage qui précède, mais le texte en est trop lacunaire pour que le sens puisse être véritablement restitué. Dans la tradition bouddhique, il existe différents types d'accomplissements. Ceux dont il est question semblent

African Studies, 28, 1984, p. 121) rapporte que dans une inscription sur pierre de Pagan datée de 1294 , il est dit que « the Buddha's Footprint of various strange marks is painted with gold and (many) colours so that it would be a marvel to look at ».

31. P. Skilling ( «Symbols on the Body, Feet, and Hands of a Buddha », Part I (Lists), JSS, 80/2, 1992, p. 67-79 ; Part II (Short Lists), JSS, 84/1, 1996, p. 5-28) met en évidence un certain nombre de traditions sanskrites (dont plusieurs sont conservées dans des traductions tibétaines et chinoises) relatives aux marques du corps du Buddha. Ces dernières sont parfois au nombre de 80 : il s'agit alors des anuvyañjana, traduits ordinairement par «marques secondaires» ou «attributs». Plusieurs de ces marques sont référencées dans les listes pāli, mais nombreuses sont également celles qui apparaissent tout à fait spécifiques aux traditions sanskrites. P. Skilling (ibid.) cite par ailleurs le Dharmapradīpikāva, texte rédigé à Ceylan à la fin du XII ${ }^{e}$ siècle, qui donne en cinghalais sanskritisé une liste des marques du Buddha. Cette liste est étudiée par E. Burnouf (Le Lotus de la Bonne Loi, Paris, 1852, réimp. 1973, p. 622-647). Elle cst de toute évidence basée sur la liste pāli. Il est à noter que la liste des 80 marques figure également dans le Dharmapradipikāva, et qu'elle est cette fois-ci formulée avec des termes pāli (cf. E. Burnouf, op. cit., p. 583-615).

32. U Mya, «A Note on the Buddha's Foot-prints in Burma », Archaeological Survey of India, Annual Report 1930-34, p. 320-331.

33. K.R. Norman, Pāli Literature, 1983, p. 157 ; O. von Hinüber, A Handbook of Pãli Literature, 1996, p. $187, \$ 407$.

34. G. Coedès, «Documents sur l'histoire politique et religieuse du Laos occidental », BEFEO, 25, 1926 , p. 10.

35. Dans une communication personnelle, S. Collins nous signale que le nom Anāgatavamsa s'applique à une famille de textes plutôt qu'à un seul. O. von Hinüber (op. cit., p. 206, $\S 448$ ) fait état d'un Anāgatavamsa particulier, cité dans le Visuddhimagga, et aujourd'hui perdu.

36. J. Filliozat, "The Commentaries to the Anaggatavamsa in the Pāli Manuscripts of the Paris Collections ", Journal of Päli Text Society, 19, 1993, p. 43-63.

37. K.R. Norman, op. cit., p. 162 ; O. von Hinüber, op. cit., p. 94, § 191.

38. G. Cœdès et C. Archaimbault, Les Trois Mondes, (PEFEO, 89), 1973. Les trois textes figurent respectivement aux $n^{\circ} 13,22$ et 14 de la liste finale (p. 256). Dans la liste de l'introduction (p. 3-4), seuls figurent le Jinālañkāra ( $\left.\mathrm{n}^{\circ} 14\right)$ et l'Anāgatavamsa $\left(\mathrm{n}^{\circ} 21\right)$. 
cependant être les accomplissements humains (manussasampatti), divins (devasampatti) et du nirvāna (nibbānasampatti), tels qu'ils apparaissent dans plusieurs textes pāli ${ }^{39}$. Il faut peut-être identifier avec le dernier la référence qui est faite dans l'inscription à l'accomplissement de Buddha (śri buddhasampatti), qui apparaît comme un stade ultime, quoique l'obtention du nirvāna ne soit pas obligatoirement réservée à un Buddha. Il est sans doute utile de relever que l'aspiration qui est formulée ici relève plutôt de conceptions cosmologiques ${ }^{40}$. Parmi les quelques mots qui subsistent dans le passage lacunaire, on peut également relever la mention du souverain cakravartin (cakravartirāja), position suprême dans l'accomplissement humain, et dont l'image est toujours fortement associée à une empreinte du Buddha avec ses 108 signes.

\section{Wat Traphang Chang Pheuak et inscription dite "Mangala"}

Si, dans la stèle du Sumanakūtaparvata, il était seulement fait référence au nombre de marques possédées par un pādalakṣana, celles-ci sont parfaitement détaillées dans deux autres inscriptions siamoises rédigées en pāli et que l'on date, en fonction de critères paléographiques, du $\mathrm{XX}^{\mathrm{e}}$ siècle de l'ère bouddhique, c'est-à-dire d'une période s'étendant entre 1357 et 1457 de l'ère chrétienne. La première est la face II de la stèle du Wat Traphang Chang Pheuak de Sukhothai, déjà étudiée par A. B. Griswold et P. na Nagara ${ }^{41}$; la seconde est une inscription sur feuille d'argent aujourd'hui conservée au musée national de Bangkok et dont la provenance a été totalement oubliée ${ }^{42}$. Nous l'appellerons par commodité l'inscription « Mangala » (P. marigala «auspicieux »), conformément au nom qui lui est donné dans les inventaires thaïlandais.

La seconde face de l'inscription du Wat Traphang Chang Pheuak décrit un buddhapāda aujourd'hui disparu. Elle est rédigée en vers, mais la langue y est souvent fautive et souffre de la comparaison avec le pāli d'autres inscriptions. La première phrase, servant d'introduction, est une citation quasiment parfaite d'un passage du Dïghanikāya contenu à la fois dans le Lakkhanasutta et dans le Mahāpadänasutta ${ }^{43}$. Il y est question de l'un (le deuxième) des trente-deux signes (lakkhana) qui caractérisent le Buddha en tant que Grand Homme (mahäpurisa) : celui-ci possède « sous la plante de ses pieds, des roues à mille rais, avec jante et moyeu, dans tous les sens bien complètes et bien divisées ${ }^{44}$. Les textes canoniques arrêtent cependant là leur description ${ }^{45}$. Pour graver à la suite de

39. Par exemple Dhp-a.III.183, Ja.I.105 et Mil.96. Cf. T.W. Rhys Davids \& W. Stede, Päli English Dictionary, p. 690.

40. Dans l'inscription du Wat Sri Chum (inscription $n^{\circ} 2$ ), la requête formulée par le prince Śrī Sadhā était d'obtenir les conditions nécessaires à l'Éveil (bodhisambhāra).

41. "Epigraphic and Historical Studies $N^{\circ} 7$ : The Inscription of Vat Trabañ Jän Phöak », JSS, 59/1, 1971, p. 157-188. Les arguments développés par les auteurs (ils font le lien avec le moine Sumana, personnage auquel des chroniques religieuses du Lanna confèrent un rôle important) pour situer la rédaction de l'inscription entre 1341 et 1347 nous paraissent extrêmement fragiles et nous ne les retiendrons donc pas. Il nous semble plus prudent de la dater de la seconde moitié du XIVe siècle.

42. Charïk Nai Prathet Thai, lem 5, Bangkok, National Library, Fine Arts Department, 1986, p. 120-130.

43. Rhys Davids, Dialogues of the Buddha, Part II, London, 1910, p. 14 ; Part III, London, 1921, p. 137 (indiqué par A. B. Griswold, p. 207). Dans le Brahmāyusutta du Majjhimanikāya, on trouvera une formulation quelque peu différente.

44. J. Boisselier, op. cit., p. 195-196.

45. Un autre signe, le sixième, revêt également une importance particulière en ce qui concerne la représentation des pieds. Comme les mains, ceux-ci sont en effet décrits comme étant (recouverts d')un réseau ou (d')un filet $(j \bar{a} l a)$. Cette précision influencera fortement les artistes, puisque c'est à l'intérieur d'un quadrillage qu'ils placeront en premier lieu les marques auspicieuses. 
cette première phrase les noms des 108 marques auspicieuses du buddhapāda, l'auteur de notre inscription a dû s'appuyer sur des textes différents, probablement la Jinālañkāra-tịk $\bar{a}$ et/ou l'Anāgatavamsa-atthakathä ${ }^{46}$.

L'inscription « Mangala » ne s'embarrasse pas de partie introductive et commence directement par la liste des lakșana. Tout comme la précédente, elle est rédigée en vers dans un pāli très incorrect. Elle présente ceci de particulier qu'elle se découpe en śloka, le dernier $p \bar{a} d a$ répétant toujours la même formule signifiant que les éléments cités précédemment «apparaissent sur le pied du Maître » (pāde dissanti satthuno). On constatera également que l'inscription est incomplète puisqu'elle ne contient que les deux premiers tiers des signes auspicieux. Nous n'avons aucune donnée susceptible de nous renseigner à propos de la suite du texte qui, par ailleurs, nécessitait peu de place ${ }^{47}$.

Il sera intéressant de confronter les listes des deux inscriptions avec celles de nos deux commentaires pāli ${ }^{48}$. À titre de curiosité, on prendra également pour référence une liste des 108 signes auspicieux telle qu'elle a été recueillie il y a quelques décennies au Cambodge par F. Bizot ${ }^{49}$. On donnera d'abord le terme pāli contenu dans la Jinālaìkāratīkā et l'Anāgatavamsa-atthakathā, en précisant les variantes de cette dernière lorsqu'elles

46. La tradition relative aux caractéristiques du second signe du mahāpurisa évolua dès les premiers commentaires. Dans la Mahāpadānasutta-ațthakathā (Sumarigalavilāsin̄̄, CDrom Chatțha Sañgāyana, 2.445) et la Brahmāyususutta-atthakathā (Papañcasūdanī, CDrom Chattha Sañgāyana, 3.375), sont respectivement énoncées 35 et 37 (la différence semble être le fait de l'édition) représentations d'objets, d'êtres, d'astres et d'entités géographiques mythiques, qui étaient toutes censées figurer à l'intérieur de la roue placée sous la plante des pieds. Nous ne savons pas selon quel processus cette liste de 35 ou 37 représentations a été élargie aux 108 que proposent la Jinālanikāra-țikā et l'Anāgatavamsa-atthakathā. Nous pouvons supposer que des textes véhiculant une tradition intermédiaire ont existé et disparu (?). La Samantakūtavannanā, rédigée au XIII' siècle, énonce près d'une cinquantaine de marigala-lakkhana entourant la roue du buddhapāda (cf. CDrom Chattha Sangāyana, vers 766-770; A. A. Hazlewood (transl.), In praise of Mount Samanta, p. 92-93). La grande majorité d'entre cux se retrouvent dans $S v$ et Ps, mais également et surtout dans Jināl-t et $A n \bar{a} g-a$. La formulation et l'ordonnance apparaissent cependant différentes. Quelques lakkhana se révèlent par ailleurs tout à fait spécifiques et ne trouvent pas d'équivalents dans les autres listes. Enfin, le chiffre total de 108 (lorsque l'on prend en compte les éléments «pluriel», comme par exemple les «six mondes divins ») n'est pas atteint, ni même mentionné. P. Skilling (communication personnelle) signale la présence, à la fois dans $S v$, Ps et Samantak, du terme $\bar{a}$ davo (《 and so on ", « etc. ») signifiant que de toute façon les listes ne sont pas closes.

On remarquera que le terme lakkhana, qui s'appliquait d'abord aux 32 signes distinctifs, est employé également pour les 108 marques. Ces dernières, parce qu'elles sont auspicieuses, sont encore appelées mangala.

47. Dans la mesure où la feuille d'argent a la forme et l'apparence d'une feuille de latanier (support habituel des manuscrits), la suite du texte aurait pu figurer au verso. Il est cependant plus probable qu'il existait une seconde feuille, aujourd'hui perdue.

48. Nous n'établirons pas de comparaison trop précise avec les données de la Sumangalavilāsin̄̄ et de la Papañcasüdani. Nous remarquerons cependant que les noms qui figurent dans ces deux textes correspondent en gros aux noms de la première moitié de notre liste, sauf macchayugala (la paire de poissons) qui figure entre vaḍhamānaka et bhaddapiṭha. Il existe également un terme - tomara (la lance) - qui n'apparaît que dans la Papañcasīiāni (entre añkusa et pāsāda). Les noms de la seconde moitié de notre liste, inspirés par des traditions cosmologiques, trouvent pratiquement tous un correspondant dans la Samantakuțavannanāa. La liste sanskritisée du Dharmapradipikāva cinghalais, donnée par E. Burnouf ( op. cit.), est pratiquement identique à la nôtre. On peut considérer qu'elle appartient à la même tradition.

49. « La figuration ... », op. cit., p. 415-416. 
existent ${ }^{50}$. On indiquera ensuite les termes figurant dans les inscriptions du Wat Traphang Chang Pheuak (TCP) et "Mangala » (Mang.) ${ }^{51}$. On relèvera enfin le terme de la liste cambodgienne (Biz.).

*1. satti : la lance.

Le terme ne figure pas dans TCP. - Il est orthographié sati (souvenir, pensée consciente) dans Mang. 1 mais est glosé comme s'il s’agissait de satī (femme chaste). - Biz. 1 : satti.

*2. sirivaccha.

Les interprétations de ce terme abondent et il est difficile de reconnaître le sens originel. Il équivaut au sanskrit śrvatsa et désigne entre autres choses la « touffe de poils d'une forme particulière sur la poitrine de Vișnu-Krșna " ${ }^{52}$. Le terme pāli vaccha désigne le veau et une traduction plus littérale pourrait être le «veau auspicieux» (?). Les gloses siamoises évoquent également la résidence (?) auspicicuse $(\dot{s} r \bar{i}-s i r i)$ ou celle de la déesse de la Fortune $(\dot{S} \mathbf{r} \bar{i}=$ Lakșmī). Comme la représentation de ce signe est souvent une femme tenant un miroir, ce dernier est également tenu pour être le sens du terme. F. Bizot parle quant à lui du popil, objet coutumier du Cambodge.

TCP 1 : sirivaccho. Mang. 2 : sirivaccho.-. Biz. 2 : sirivaccho.

*3. nandivāvatta : tournant auspicieusement (vers la droite), auspicieux.

Dans la glose siamoise, il est fait référence à une fleur (peut-être le gardénia) ou à un objet en forme de fleur. Il s'agit en fait de l'équivalent pāli du sanskrit nandyāvarta, « sorte de diagramme ; objet qui en a la forme ${ }^{53}$.

TCP 3: nandivatta (cercle du plaisir?). - Mang. 3: nandiyā (le composé est amputé et donc manifestement incompris). - Biz. 3 : nandiyavattamey (la fleur Malatī).

*4. sovatthika $=$ skt. svastika.

Il s'agit d'une $"$ sorte de croix mystique servant de signe auspicieux ${ }^{54}$ (en l'occurrence, de la croix gainmée).

TCP 2 : sovatthi. - Mang. 4 : suvatthiko. - Biz. 4 : sovatthiko.

*5. vatainsaka: (cf. vatarisa) : « a kind of head ornament, perhaps ear-ring or garland worn round the forehead $\gg$ 5.

TCP 4 : vatamsakam. - Mang. 5 : vattamsako. - Biz. 5 : vatamso (le turban).

*6. vaḍ̂hamāna (nt.) : le vase ${ }^{56}$.

TCP $8:$ vuddhamānakam. - Mang. 6 : bhaddhamāno - Biz. 6 : vaḍ̣hamanam (le Pāyasī).

*7. bhaddapitha: le trône.

C'est peut-être ce terme qui figure dans TCP 7 (le texte est lacunaire à cet endroit). - Mang. 7 : hhaddapitham. Biz. 7 : pithakam.

*8. ankusa : l'aiguillon (à éléphant).

TCP 5 : arkuso. - Mang. 9 : aikkuso. - Biz. 8 : arikuso.

*9. pāsāda: le palais.

TCP 6 : pāsādo. - Mang. 8 : pāsāado. - Biz. 9 : pāsādo.

50. N'ayant pas eu accès directement au texte de ces deux ouvrages, nous nous sommes basé pour la Jināl-t (dont il existe une édition birmane) sur la liste que donne la traduction en thaï ( Roy Phra Phutthabat Samay Tang Tang ", in Phutthabat Laksana Lè Roy Phra Phutthabat Nai Prathet Thai, p. 36-43) de l'article de W. C. Sailer, «Chronology of Buddha Footprints », ibid., p. 27-35, la première respectant en effet l'alphabet pāli alors que le second ne restitue pas les signes diacritiques - et pour l'Anāg-a sur les indications données par U Mya et reprises par A. B. Griswold et P. na Nagara.

51. On indiquera en premier lieu le terme sans déclinaison, tel qu'il est répertorié dans les dictionnaires. TCP et Mang. l'orthographient cependant dans sa valeur casuelle nominative et restituent par exemple pour les noms masculins et neutres se terminant en «a » les déclinaisons respectives «o » et «am ».

52. N. Stchoupak, L. Nitti et L. Renou, Dictionnaire sanskrit-français, p. 743.

53. Ibid., p. 350 . Ce symbole a été l'objet d'un certain nombre d'articles spécialisés, cf. P. Skilling, «Symbols ... », Part I, op. cit., p. 78, note 52. Son identification ne semble toujours pas assurée.

54. Ibid., p. 881.

55. T.W. Rhys Davids, William Stede, Pāli-English Dictionary, p. 594.

56. Communication personnelle du Prof. O. von Hinüber. 
* 10. torana : le portail.

Le terme est oublié dans TCP. - Mang. 10 : toranam. - Biz. 10 : toranam.

*11. setacchatta : le parasol blanc, emblème de la royauté.

TCP 9 : setachattam. - Mang. 11 : setachattam. - Biz. 11 : chattamev.

*12. khagga: 1'épée, un des insignes royaux.

TCP 10 : khaggo.-- Mang. 12 : khaggan!. - Biz. 12 : khaggo.

*13. tălavanta : l’éventail (en feuilles de palmier).

TCP 11 : tälavantam. - Mang. ne mentionne pas le terme. -- Biz. 13 : talapannam (désigne la feuille de palmier).

*14. malürahattha: (litt. main du paon) l'éventail en plume de paon. - Anāg-a se différencie de Jināl-t en donnant morapattam (nt.) : plume de paon (mora est la forme contractée de mayüra).

TCP 13 : morahatthakam. - Mang. 13 : morahattho. - Biz. 14 : morapiñchakavījanī (l'éventail en plumes de queue de paon). Il semble que le composé ait été fabriqué à partir d'un élément sémantique (vijani = éventail) appartenant en fait au terme suivant.

*15. cāmara: la queue d'un yak utilisée comme un plumeau ou un chasse-mouche. - Le terme est remplacé dans $A n \bar{a} g-a$ par vālavijañ̄ qui désigne un éventail fait avec la queue d'un yak.

TCP 12 : vijinī. Mang. $17:$ vălavịjañ. - Biz. (vījanī semble avoir servi à préciser le terme $\mathrm{n}^{\circ}$ 14).

*16. umhissa (nt.) : le turban.

TCP 14 : umhisam. - Mang. $14:$ unhiso. - Biz. $15:$ unhiso.

*17. mani : le joyau.

TCP $17:$ mani. - Mang. $16:$ mani. - Le terme manque dans Biz.

*18. patta: le bol.

TCP 15 : patto. - Le terme manque dans Mang. ; en $15^{\mathrm{c}}$ position, celui-ci donne ratanavalliyo (?). Il s'agit peut-être du joyau veluriya ou d'un joyau en ornement d'oreille (ratanavallika). - Biz. 16 : patto.

* 19 : sumanadāma (nt.) : la guirlande de jasmin.

TCP 16 : dāma. - Mang. 17 : sumanadāmam. - Biz. 18 : sumanadamaṇ.

*20: niluppala : le lotus ou lis d'eau bleu.

TCP 18-19 cite le lotus (uppalam) mais le fait suivre d'un dvandva où les deux adjectifs bleu (nìla) et rouge (ratta) copulent. - Mang. 21 : niluppalam .-Biz. 19 : nìluppalam.

*21 : rattuppala : le lotus ou lis d'eau rouge.

TCP 18-19: cf. remarque précédente. - Mang. 20 : rattuppala. - Biz. 20-21 : rattasetuppalam (les deux adjectifs rouge et blanc forment un composé).

*22 : rattapaduma (nt.) : le lotus rouge. - Anāg-a diffère en ne citant pas la catégorie des ${ }^{\circ}$ paduma mais en ajoutant setuppala (le lotus ou lis d'eau blanc) à la catégorie des 'uppala.

TCP 20 : padumam (il n'est question que d'un seul paduma). - Mang. 19 : paduma (il n'est question que d'un seul paduma).--Biz. 22 : padımam (il n'est question que d'un seul paduma).

*23 : setapaduma : le lotus blanc. - Anāg-a : cf. remarque précédente.

TCP : cf. remarque précédente. - Mang. 22: setupala (comme Anāg-a). - Biz. 21 : setuppalam (comme Anāg-a).

*24 : pundarika (nt.) : le lotus blanc. - Anäg-a donne rattapundharika (sic) et setapundharika (le lotus blanc-blanc).

TCP : le terme ne figure pas. - Mang. 23 : pundarikă. - Biz. 23 : pundari.

Conclusion : TCP ne compte que trois références à des lotus ( 2 uppala et 1 paduma). Mang. et Biz. comptent cinq références ( 1 padıma, 3 uppala et 1 puṇạikika).

*25 : puṇnakalasa : le pot à eau rempli. - Anāg-a donne puṇnaghaṭa (pichet ou pot rempli).

TCP 23 : punnaghato. - Mang. 24 : punoghato. - Biz. 24 : punnghato.

*26 : pumn!napatta (nt.) : le bol rempli. - Anāg-a donne punnapāti (f.) : le bol rempli.

TCP 22 : pumnapāti. - Mang. 25 : puṇnacāti (l'orthographe correcte est punnacăta : la jarre ou le pot rempli(e)), - Biz. 24 : puṇnacați 
*27 : samudda : l'océan.

TCP 21 : scumuddo. Mang. 26-29 : samuddācaturo (les quatre océans). - Biz. 26 : samuddo.

*28: cakkavālapabbata: les montagnes qui encerclent l'univers. - Anāg-a donne simplement cakkavaila: le monde, la sphère.

TCP 25 : cakkaràlà. - Mang. 30 : cakkavālo. - Biz. 27 : cakkavalakam.

*29 : himarantapabhata : la montagne himavā (Himālaya). - Anāg-a donne seulement himavā.

TCP 24 : himavă. - Mang. 31 : himavă. - Biz. 28 : hemav'a.

*30 : merupabhata : le mont Meru. - Anāg-a donne simplement meru.

TCP 27 : meru. - Mang. 32 : neru. - Biz. 9 : sineru.

*31 : surịamandala : le globe du soleil. - Anāg-a indique seulement sīrịa (sic).

TCP 28 : surịa. Mang. 33 : surịo. - Biz. 30 : surịo.

*32 : candamandala : le globe de la lune. - Anāg-a indique candimā : la lune.

TCP 29 : candimā. - Mang. 34 : candimāsa. - Biz. 31 : candim.

*33 : nakkhatta (nt.) : les constellations.

TCP 26 : nakhattā. - Mang. 35 : nakkhattako. - Biz. 32 : nakkhattā.

*34-37 : saparivāracatumahādipā : les quatre grands continents avec ce qui les entoure.

TCP 30-34 : caturomahādīpādisahassaparittakā (les quatre grands continents et les deux mille (îles) inférieures). - Mang. 36-40 : cattaro ca mahādīpa (...) sahadvisahassādipparivārā (étant donné la forme très incorrecte de la seconde partie, il n'est pas sûre que celle-ci ait été bien comprise). - Biz. 33-37 : caturo dip disahassaparittakā.

Pour Jināl-t, il est évident que l'on compte quatre unités. Pour TCP, Mang. et Biz., par contre, il semble qu'il faille mettre l'ensemble constitué par les 2000 îles inférieures à part et donc compter 5 unités.

*38: saparîārasattaratanasamaingicakkavatti: le monarque universel avec ses sept trésors et son entourage (armée). - Anāg-a indique seulement sapariso cakkavatti : le Monarque universel avec son asscmblée.

TCP 36 : saparisocakkavatti. - Mang. 44 : cakkavatimahāıājā senāya parivārito (le grand roi cakkarattin entouré de son armée). -- Biz. 38 : cakkavatti.

*39 : dakkhināiattasetasaikha: la conque blanche dont les volutes tournent vers la droite.

TCP 35 : dakkhinävattasankho. - Mang. 45 : dakkhināvattasakho. - Biz. 39 : vattasankho.

*40 : suran!mamacchayugala : la paire de poissons en or.

TCP 37 : yamakam hemamaccham (la paire de poissons en or). - Mang. 46 : swannamaccha luggalam. - Biz. 40 : macchānam vugalam.

*41 : cakkāindha: le disque utilisé comme une arme. - Anäg-a indique seulement cakka: le disque ou la roue.

TCP 38 : cakka. - Mang. 47 : cakkam. - Le terme ne figure pas dans Biz.

*42-48 : sattamahāgangā : les sept grandes rivières.

TCP 41-47 : sattagaingā. - Mang. 48-54 : sattamahānadī (les sept grandes rivières). - Biz. 41-47 : satt mahāganigā.

*49-55 : sattakulapabbata: les sept groupes de montagnes. - Anāg-a indique sattamahāselā : les sept grandes montagnes.

TCP 48-54 : mahāselā. - Mang. 62-68 : mahākulabalāsatta (?). - Biz. 48-54 : satt mahāselā.

*56-62 : sattasidantasāgarā : les sept océans siddanta. - Anāg-a indique sattamahāsarā.

TCP 55-61 : sattamahāsarā. - Mang. 55-61 : sattamahāsarāa. - Biz. 55-61 : satt mahāsarā.

*63 : suran!̣asupannaräaja: roi Garuda en or.

Le terme ne figure pas dans TCP. - Il est remplacé dans Mang. 69 par pakkhirājā : le roi ailé. Peutêtre faut-il lier à ce composé la référence uttamo (le meilleur) qui suit directement. - Biz. 62 : supan!?o. 
*64 : samsumāra (ou sumsumāra) : le crocodile ${ }^{57}$.

TCP 63 : sumsu. - Mang. 71 : susumāra. - Biz. 63 : samsumāro.

Dans TCP 40 , il était déjà fait référence à un crocodile kumbhilo que l'on ne retrouve nulle part ailleurs dans les listes. Sous celui-ci, se cache peut-être la référence au roi Garuḍa qui nous manquait précédemment.

*65 : dhajapatāka: le drapeau et la bannière (les deux se différencient apparemment par leur système d'attache). Bien que représentant deux objets, ils semblent avoir dans la liste une valeur au singulier.

TCP 39 : dhajja et TCP 62 : patākko. - Mang. 73 : dhajjo. - Biz. 64 : dhajapatākam.

*66 : suvannasivikā (f.) : le palanquin en or. - Anāg-a indique ratanapātañki. Si ratana (joyau) ne pose pas de problème à la traduction, il en va autrement avec pātańkī que le Pāli-English Dictionary (p. 450) pense être, en y mettant un point d'interrogation, une chaise à porteurs. Le rapport avec pallarikam est également évoqué. Il est intéressant de constater que le terme pallañko (trône) figure dans Mang. 72 et Biz. 17 et fait donc peut-être double emploi avec pātañki.

TCP 64 : pātañki (pātaniki). - Mang. 74 : rattanapātañki. - Biz. 65 : pātañkī.

*67 : suvannavalavījanī : l'éventail (fait avec la queue d'un yak) en or.

TCP 65 : siuvannaválabijanī. - Mang. 76 : cāmaro (la queue d'un yak utilisée comme un chassemouche). Il est intéressant de constater que le choix des mots est inversé par rapport au $n^{\circ} 15=$ Mang. 17. - Biz. 66 : valavijani.

*68: kelāsapabbata : la montagne Kelāsa.

TCP 70 : keläsapabbato. - Mang. 75 : kelaso. - Biz. 67 : kelāsapabvato.

*69 : sihharajjā : le roi des lions.

TCP 66 : suvannasīha (le lion d'or). - Mang. 77 : siharājā. - Biz. 68 : sīharājā.

*70 : bvaggharajāa : le roi des tigres.

TCP 67 : braggho (entrant en composé avec sīha, il bénéficie aussi de l'adjectif suvanna: or). Mang. 79 : hyaggharājā. - Biz. 69 : byaggharājā.

*71 : valāhaka-assarājāa : le roi des chevaux (de la famille) Valāhaka. - Anāg-a indique valāhoassarāja.

TCP 68 : valaho-assa. - Le terme manque dans Mang. car l'inscription s'interrompt à ce moment-là. - Biz. 70 : valăhavako.

*72 : uposathachaddantahatthirājā : le roi des éléphants Uposatha et Chaddanta. - Anāg-a indique uposotharäranarājā : le roi des éléphants Uposatha.

TCP 69 : uposathovärana. Le texte précise à propos des quatre animaux qu'«ils sont tous appelés par le mot roi " (sabbe te rājasaddena avhavāa). - Biz. 71 : uposatho, chaddanto. Le deuxième, considéré comme un autre signe, est sans doute à replacer aux côtés du premier pour ne former qu'un seul ensemble. Il est cependant vrai qu'il existe un roi des éléphants Uposatha et un roi des éléphants Chaddanta.

*73 : vāsukināgarājāa: Vāsukī, roi des nāga. -- Anāg-a indique bāsuki-uragarājāa : Bāsukì, roi des serpents.

TCP 73 : vāsuki. - Biz. 72 : vasukī nāgo.

*74: hamsarāja : le roi des hamsa.

TCP 71 : hanso. - Biz. 73 : hamso.

*75 : usabharājā : le roi des taureaux.

Peut-être TCP 77 ou 78 qui sont lacunaires. - Biz. $74:$ usapho.

*76 : erāvanahatthirājāa : le roi des éléphants Erāvaṇa. - Anāg-a indique eravāno nāgarājāa : le roi des éléphants Erāvana (le terme polysémantique năga est plutôt employé pour désigner l'éléphant dans $A n \bar{a} g-a$ et pour désigner le serpent dans Jinäl-t $)$.

TCP 74 : erāuano. - Biz. 75 : eravano.

57. Le Prof. von Hinüber évoque un animal appartenant à la catégorie des dauphins: le «Schnabeldelphin ». 
*77 : suvannamakara : le makara en or.

Biz. 76: marikaro. - le terme n'apparaît pas dans TCP où l'on note par contre, en position 76 , suramnabhamaro: l'abeille d'or; bhamaro apparaît dans Biz. 77 ; il est par contre absent dans Jinäli $t$ et Mang.

*78: catumukhasman!̣anāvā : le bateau d'or «à quatre faces » (?). - Anāg-a indique catumukhan $\bar{\imath}$ sovannanāià.

Biz. 80 : harināiva catummukhā. - Cette référence est comptée pour deux avec TCP $81:$ harināvā, le bateau d'or, et TCP 82 : catummukhā, quatre faces (supposées être celles de Brahma). C'est cette dernière interprétation, plus logique, qui doit être correcte.

*79 : savacchakadhemu: la vache et son veau.

Biz. 81 : savacchakā gālō. - Il n'est pas fait mention de cette référence dans TCP.

*80 : kimpurisa : homme-oiseau. - Anāg-a indique kinnara : homme-oiseau.

TCP 83: kinnaro (pakkhi). -- Mang. 42 : kinnara. - Biz. 82 : kinnaro.

*81 : kinnar $\bar{l}$ : femme-oiseau.

TCP 84 : kinnarit. - Mang. 43 : kinnarȳyo. - Biz. 83 : kinnarī.

*82 : karavika: le coucou (le mahāpurisa parle avec l'intonation du coucou).

TCP 75 : karaviko. - Biz. 84 : karaviko.

*83 : mavurarājā : le roi des paons.

Le terme ne figure pas dans TCP, à moins qu'il ne faille le confondre avec kukkusüro $($ sic $)=k u k k u t a$ (coq) ? - Biz. 85 : mayluro.

*84: koñcarāja : le roi des grues.

TCP 80 : koñco. - Biz. 86 : koñcarājāa.

*85 : cakkaräkarajā : le roi des oies rousses.

TCP 72 : cākavāko. - Biz. 87 : cakkavākadijo.

*86 : jīnañjīvakarājā : roi d'une catégorie d'oiseau, sorte de faisan ou de perdrix. - Anāg-a indique jūiajìvakarājā.

TCP 85 : jīinañjīiakanāmakā (du nom de jīvañjīiaka). - Biz. 88 : jūvañjīvakanāmakā.

*87-92 : chadevaloka : les six mondes des Deva. - Anāg-a indique chakāmāvacaradevalokā : les six mondes divins du domaine du Désir.

TCP 86-91 : chakāmāvacarālokā. - Biz. 89-94 : chakkāmāvacarādevā.

*93-108 : solasabrahmalokā : les seize mondes des Brahma.

TCP 92-107: brahmalokā solasa. - Biz. 69 : hrahmalokā solas.

Du point de vue purement numérique, nous remarquons que si Jināl-t et Anāg-a présentent bien une liste de 108 éléments, il en va quelque peu différemment avec TCP et Biz. La première ne compte en effet que 107 éléments auspicieux et il y manque peut-être un élément tel que torana $\left(\mathrm{n}^{\circ} 10\right)$ ou savacchakadhenu $\left(\mathrm{n}^{\circ} 79\right)$. La seconde est excédentaire puisqu'elle donne 110 marques dont une, suvannakacchapa (la tortue d'or), lui est spécifique, alors que d'autres, telles kukkuta (le coq) et pallañka, se retrouvent respectivement dans TCP et Mang. Ces dernières répètent d'ailleurs sans doute, sous un nom différent, des marques qui figuraient déjà. Quant à la liste de Mang., nous avons remarqué qu'elle contenait 79 lakṣana, c'est-à-dire qu'il ne lui manque que 5 éléments si l'on met à part les 16 mondes des Brahma et les 6 mondes des Deva qui forment déjà à eux seuls un ensemble important.

Du point de vue sémantique, on remarque que mis à part quelques menus problèmes de concordance, toutes nos listes correspondent d'une façon générale et respectent en gros un certain ordre. On remarquera par exemple que les vingt-six premiers lakșana désignent plutôt les attributs d'un souverain, alors que les quarante-deux suivants ressortissent plutôt à la cosmologie et à la géographie sacrée. Quant au dernier tiers de nos listes, il est consacré aux animaux mythiques puis à vingt-quatre des étages supérieurs. À ce niveau de l'étude, on peut donc accepter sans trop tergiverser que nos inscriptions soient inspirées 
par les textes para-canoniques auxquels nous avons fait référence, ou tout au moins par une tradition qui leur était très proche. Si l'on pousse l'analyse plus loin et que l'on considère la question de la formulation, d'autres données, plus précises, viennent appuyer ces premières conclusions. On remarque d'abord que TCP et Mang. respectent plutôt la terminologie employée par Anāg-a lorsque celle-ci diffère de Jināl-t. Il semble donc bien que ce soit plutôt ce premier texte qui ait servi de référence ${ }^{58}$. On constate encore que nos deux inscriptions, bien que datant sans doute toutes deux du XIV ${ }^{\mathfrak{e}}$ ou de la première moitié $\mathrm{du} \mathrm{XV}^{\mathfrak{e}}$ siècle, présentent entre elles des différences qui sont significatives et furent donc rédigées de façon indépendante, à partir de modèles différents.

\section{Les buddhapāda inscrits}

À côté des inscriptions décrivant d'une façon plus ou moins détaillée des buddhapāda, nous avons également cinq empreintes (dont une double) du Buddha qui sont elles-mêmes inscrites et apportent donc un témoignage encore plus direct. Toutes sont datées du $\mathrm{XX}^{\mathrm{e}}$ siècle de l'ère bouddhique $(=1357-1457)$ et sont pour l'instant rattachées à la culture de Sukhothai, voire aux débuts de celle d'Ayuthya. Etant donné les différences importantes qui les opposent, il est nécessaire de les étudier séparément.

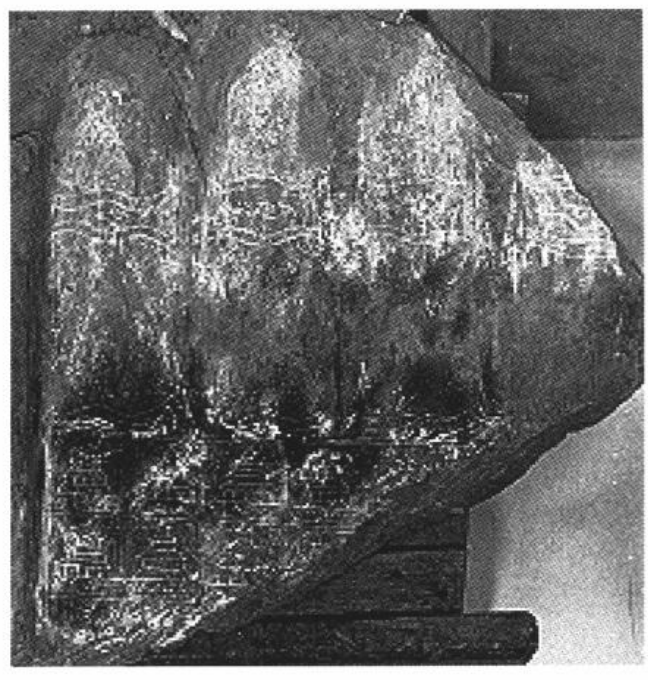

Buddhapāda du Wat Si Chum (Sukhothai)

(Fine Arts Department)

\section{Le buddhapāda du Wat Si Chum}

Le premier de ces buddhapäda inscrits a été retrouvé au Wat Si Chum à Sukhothai ${ }^{59}$. Il est très fortement endommagé puisqu'il ne subsiste qu'une moitié transversale de la partie supérieure (c'est-à-dire quatre orteils et leur base) et par là-même des cartouches inscrits que surmonte à chaque fois un dessin représentant un prāsāada. Les études qui ont déjà été faites sur les buddhapāda, notamment ceux de Birmanie, montrent que la figuration de séries de prāsāda représente toujours les mondes des Brahma et des Deva,

58. On formulera toutefois cette conclusion avec prudence. L'édition critique d'éventuelles versions des deux textes conservées en Thaïlande n'a en effet toujours pas été faite.

59. Chariik Nai Prathet Thai, lem 5, p. 43-46. La pièce est conservée dans un dépôt du musée de Sukhothai qui n'est pas accessible au public. 
c'est-à-dire les vingt-deux dernières de nos 108 marques auspicieuses. Il arrive que cellesci soient placées comme ici dans la partie la plus haute de l'empreinte, conformément à leur position éminente dans les représentations cosmologiques, et relèguent les autres marques relatives aux hommes ou au monde terrestre à un niveau inférieur, c'est-à-dire à celui du talon ${ }^{60}$. Le graphisme est uniforme et se caractérise par la répétition d'un même motif, de telle sorte qu'il est impossible de distinguer non seulement les différents mondes des Brahma entre eux, mais également ceux-ci des mondes des Deva. Dans la mythologie bouddhique, il est cependant admis que les premiers occupent la position suprême au sein des mondes célestes. Cette conception trouve parfois sa traduction dans l'organisation des motifs : il arrive en effet que ceux-ci soient répartis en séries horizontales de huit et il devient alors facile d'identifier deux séries supérieures de prāsāda, lorsqu'elles sont complètes, avec les mondes des Brahma. Le fait est confirmé par le buddhapāda du Wat Si Chum qui offre en outre cette particularité importante d'être (à notre connaissance) le seul dont les motifs soient précisés par une légende. Les sept cartouches dont nous pouvons encore lire le texte sont répartis sur deux bandes et donnent chacun un nom des seize mondes des Brahma. Nous pouvons ainsi lire à la suite :

1. pārisajjā / purohitā / mahābrahmā / vehapphalā /........

2. parittābhā / appamānāābhā / ābhassarā /...

Il est utile à ce stade de relever un problème tenant à la proportionnalité de l'empreinte. La partie de la pierre qui subsiste ne révèle en effet d'une façon sûre que le dessin de quatre orteils. Le cinquième n'est pas visible mais il ne fait pas de doute qu'il existait et l'on peut donc, en fonction de la largeur pratiquement équivalente des quatre premiers orteils, restituer la largeur totale de l'empreinte. Si l'on prend toutefois en compte la largeur relativement égale des cartouches qui subsistent, on en vient à déduire que chacune de nos deux bandes comportait non pas huit cartouches (ce qui représentait un total de seize noms), mais apparemment neuf, ou tout au moins laissait de la place pour un cartouche supplémentaire ${ }^{61}$. Cete conclusion doit cependant être formulée avec une certaine prudence : nous ne pouvons en effet être sûr de la largeur des cartouches que pour neuf d'entre eux. Rien n'indique que les autres aient été traités absolument de la même manière.

Des précisions s'imposent sans doute lorsque l'on évoque les seize mondes des Brahma et les six mondes des Deva. Il faut remarquer d'abord que si ceux-ci sont mentionnés dans la Jinālarikāra-țikā et l'Anāgatavamsa-ațhakathāa, ils sont toujours considérés en tant qu'ensemble et donc jamais en tant qu'unités différenciées. Ce fait explique sans doute l'uniformité des représentations. La référence à ces mondes est cependant courante dans d'autres textes, y compris ceux relevant du canon, et nombre de détails sont alors donnés au sujet de leur composition. On peut donc imaginer que l'auteur des légendes de notre buddhapāda avait en tête l'une de ces traditions littéraires lorsqu'il accomplit son œuvre.

Le nombre des mondes de Brahma n'est habituellement pas fixé à seize mais à vingt ${ }^{62}$. On considère en effet que ces derniers se répartissent sur deux niveaux : le monde matériel

60. Deux conceptions s'opposent ici. Dans la plupart des buddhapāda de Pagan, les prāsāda sont représentés au centre, ou plus justement autour de la roue qui est figurée comme une fleur. Conformément aux conceptions indiennes, il s'agit là également d'une position éminente.

61. Les buddhapāda du Wat Traphang Thong, du Khau Kôp, du Khau Nang Thong et du Wat Sri Khom Kham (Phayao) - ainsi que plusieurs empreintes de Pagan - comportent également neuf divisions dans leur largeur. Les signes auspicieux qui les occupent ne sont cependant pas les mondes des Brahma et des Deva.

62. G. P. Malalasekera, Dictionary of Pāli Proper Names, vol. II, p. 336 ; J. R. Haldar, Early Buddhist Mythology, p. 30 et p. 44 note 147 . 
ou celui des apparences (Rūpaloka), divisé en seize étages, et le monde immatériel ou de l'absence d'apparences (Arüpaloka), divisé en quatre étages. Logiquement, dans la mesure où il n'est pas représentable, le monde immatériel n'a pas sa place parmi les signes auspicieux, et c'est sans doute la raison qui explique son absence dans la Jinālañkāra-tīkā et l'Anāgatavamsa-atthakathā. Il semble cependant que certains artistes birmans n'aient pas été effrayés par le paradoxe dans la mesure où l'on trouve parfois à Pagan la représentation de vingt mondes des Brahma ${ }^{63}$. Il est utile de remarquer que dans la mythologie sarvāstivādin, la liste du Rüpaloka (ou Rūpāvacara) comporte deux noms supplémentaires - ceux des Anabhraka et des Punyaprasava - portant ainsi le nombre des étages à dix-huit ${ }^{64}$. Il serait cependant imprudent, à ce stade de l'analyse, de tirer des conclusions trop rapides au sujet de la largeur du buddhapāda qui nous concerne.

Des seize cartouches représentés d'une façon sûre à l'origine, il n'en subsiste que dix de façon visible et sept où les noms des mondes apparaissent encore. Il est intéressant de remarquer que par rapport à l'ordre retenu dans les traités de cosmologie, la notation des noms sur l'empreinte ne respecte pas un ordre totalement linéaire. Si les trois premiers cartouches du bandeau supérieur donnent en effet les noms des trois premiers mondes du Brahmaloka - (Brahma)pārisajja, (Brahma)purohita, Mahābrahma -, le quatrième donne le nom du dixième monde - Vehapphala - et il faut en fait descendre au bandeau inférieur pour découvrir les noms des quatrième, cinquième et sixième mondes: Parittābha, Appamānā et Ābhassara. Chacun des seize mondes étant, selon la littérature normative, associé à des états psychiques, il existe par ailleurs une nouvelle répartition en quatre étages principaux correspondant chacun à un degré de méditation (jhāna). Les trois premiers mondes (1-3) se regroupent alors dans un plan que l'on nomme Pathamajjhāna (premier jhāna) ; les trois suivants (4-6) font partie du Dutiyajjhāna (deuxième jhāna) ; les trois qui leur succèdent (7-9) sont inclus dans le Tatiyajjhāna (troisième jhāna) et les sept derniers (10-16), enfin, sont compris dans le Cätutthajjhāna (quatrième jhāna). Dans notre empreinte, nous avons donc de façon apparente un premier bandeau où se trouve le Pathamajjhāna, puis le premier élément ( $10^{\mathrm{c}}$ monde) du Cätutthajjhāna. Il ne fait alors pratiquement aucun doute que les éléments qui suivent (non visibles) font partie de ce dernier plan et représentent les $11^{\mathrm{c}}$ (Asaññasatta), $12^{\mathrm{c}}$ (Aviha), $13^{\mathrm{c}}$ (Atappa) et $14^{\mathrm{c}}$ (Sudassa) mondes. Le deuxième bandeau commence, comme nous l'avons dit, par les éléments du Dutiyajjhāna $\left(4^{\mathrm{e}}, 5^{\mathrm{e}}\right.$ et $6^{\mathrm{e}}$ étages). Il est logique, étant donné ce qui a déjà été constaté, qu'il se poursuive par les éléments (non visibles) du Tatiyajjhāna $\left(7^{\mathrm{c}}, 8^{\mathrm{c}}\right.$ et $9^{\mathrm{c}}$ étages) et qu'il se termine par les deux derniers éléments du Cātutthajjhāna $\left(15^{\mathrm{c}}\right.$ et $16^{\mathrm{e}}$ étages) qui se situent alors juste en dessous des $13^{\mathrm{C}}$ et $14^{\mathrm{C}}$ étages ${ }^{65}$. Dans l'agencement des cartouches, il y a ainsi une nouvelle logique qui permet d'ordonner d'une façon satisfaisante les éléments impairs d'un ensemble pair.

La partie qui subsiste de l'empreinte ne permet en rien de certifier que le monde des Deva ait été représenté. En dessous de nos deux rangées consacrées au Brahmaloka, n'est visible en effet qu'un bandeau de transition figurant de petits cercles ornementaux. Il serait cependant tout à fait logique que d'autres motifs, et donc d'autres signes auspicieux, aient figuré.

63. G. H. Luce, op. cit., p. 245.

64. L. Renou et J. Filliozat, L'Inde classique, vol. II, Paris, 1996 (rééd.), p. 526.

65. Les quatre derniers noms forment ainsi un carré et se distinguent des autres noms agencés d'une façon linéaire. Cette particularité peut être pertinente. Dans la tradition pāli, Aviha, Atappa, Sudassa, Sudassin et Akanițtha sont des divisions de l'étage appelé Suddhāvāsa, ce dernier étant associé aux étages Vehapphala et Asaññasatta (nos étages 11 et 12), formant ainsi un ensemble de trois, comme les autres jhāna (cf. L'Inde classique, p. 526). 


\section{Le buddhapāda du Wat Sadet}

Le second buddhapāda inscrit est celui du Wat Sadet de Kampheng Phet ${ }^{66}$. Conservé aujourd'hui au musée national de Bangkok, il consiste en une lourde plaque de métal rectangulaire dont deux grands angles ont disparu. L'empreinte proprement dite comporte en son centre un grand cakra divisé en plusieurs cercles concentriques. Ces derniers contiennent la plupart des 108 signes auspicieux alors que les prāsāda, occupant une place à part, sont posés sur le bord extérieur de la rouc. Trois grandes bandes sur lesquelles sont gravées des séries de personnages encadrent encore le buddhapāda. L'une est verticale et se divise en cinq panneaux où se répartissent, différenciées par leur taille et par leur posture, huit figures dont les noms sont inscrits. Les deux autres bandes sont horizontales et reproduisent chacune, à une échelle différente, une rangée de personnages debout qu'une inscription linéaire permet également d'identifier. Le dessin est stéréotypé. Un premier coup d'œil permet d'assurer que la bande supérieure représente des Buddha, alors que la bande inférieure figure des moines dont on montre alternativement la face et le profil. Les deux bandes (ainsi que les inscriptions) se révèlent cependant incomplètes en raison de la cassure des deux angles du côté droit. Une troisième ligne inscrite horizontale, placée sur un des rebords de l'empreinte, offre par ailleurs des informations sur la donation même de l'objet. Nous étudierons chacune de ces inscriptions séparément.

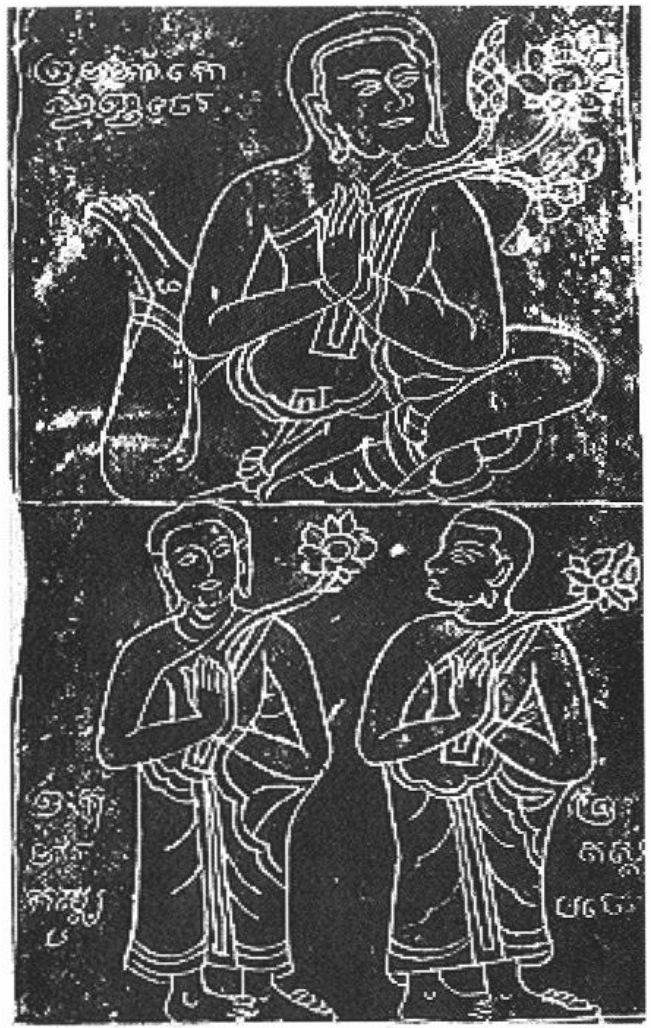

Détail du huddhapāda du Wat Sadet (musée national de Bangkok) 
Ce qui subsiste de la ligne inscrite de la bande horizontale inférieure montre une suite de vingt noms dont dix-neuf au moins désignent des grands disciples ayant atteint l'état d'arahant. Il s'agit dans l'ordre de Sāgato (Sāgata Thera), Sugato (Sugandha Thera ?), Sobhaddo (probablement Subhadda Thera), Pabhankara (Thera), Samiddhi (Thera), Loluddhāyī (Lāludāyi Thera), Kạludāyī (Thera), Udāyī (Thera), Gavampati (Thera), Simbalī (Sivvalī Thera), Upālī (Thera), Bhaddraji (Bhaddaji Thera), Assaji (Thera), Vakkali (Thera), Punnathera, Bharādvāja (Thera), Subhūta (Thera) ${ }^{67}$, Añgulimāla (Thera), Nāgathera (Nägapupphiya Thera ou Nāgasamāla Thera ?) et Rațthapāla (Thera). La liste est forcément incomplète en raison de l'état du buddhapāda. On peut établir, sans trop de risque de se tromper, qu'il s'agit là d'une référence aux quatre-vingts principaux disciples (asīti mahā sāvakāa) du Buddha, et que les trois quarts de l'inscription et des représentations qui l'accompagnaient ont donc disparu. Les vingt noms figurent en effet à la suite (dans un ordre inversé) dans un "khāthā ${ }^{68}$ » du nord de la Thaïlande qui en compte soixante autres ${ }^{69}$. Cette liste des quatre-vingts disciples apparaît cependant marginale : il en existe (au moins) une seconde - tout à fait distincte ${ }^{70}$ - dont nous verrons plus loin qu'elle est également en rapport avec un buddhapāda. C'est cette seconde liste qui est d'ailleurs normative, pour ne pas dire orthodoxe, dans la mesure où c'est elle seule qui est donnée dans les publications relatives aux grands disciples ${ }^{71}$. Elle tire en effet sa légitimité du fait qu'elle est mentionnée intégralement et exclusivement dans la Paramatthadipanī, œuvre de Dhammapāla qui contient entre autres commentaires celui des Theragătha $\bar{a}{ }^{72}$. La première liste, par contre, ne semble révéler d'exemples qu'à un niveau très régional et pour l'instant spécifiquement thaï ${ }^{73}$. Probablement ignorée (voire rejetée) par la hiérarchie bouddhique attachée au respect du canon cinghalais, il n'en reste pas moins qu'elle s'appuie elle aussi sur le corpus pāli ${ }^{74}$. Il sera donc sans doute utile d'essayer de déterminer à quelles sources originales elle vint puiser. L'inscription du buddhapāda du

67. Les noms de Bhāradvāja et de Subhūta sont précédés du terme respectueux «Phra ».

68. Transcription thaïe du pāli gāthā. Le terme n'a cependant pas ici le sens habituel de « vers » ou de "stance ». Il désigne une formule composée de mots ou de syllabes à laquelle on prête des vertus magiques. Il est donc apparenté au mantra et les deux termes sont souvent interchangeables. On donnera à la trancription « khāthā » le genre masculin.

69. «Asītisāvakakhāthā », référencé également sous le titre «Koṇ̣añño », in Suat Mon Muang Neua, Chiang Mai, 2537 (1994), p. 182. L'orthographe est souvent corrompue et rend difficile l'identification d'une quinzaine de noms. Le lettré qui s'est occupé de l'édition de l'ouvrage n'a pas pu nous renseigner sur l'origine de ce «khāthā " particulier. Les vingt noms qui nous concernent sont mentionnés à la suite depuis Rațthapālo ( $9^{c}$ position) jusqu'à Sāgato (29* position). Une exception cependant : le nom « Pāvalī (?) » qui figure à la 24' position dans l'ouvrage n'est pas mentionné dans notre buddhapāda.

70. Cf. Phra Thammapidok (Prayuth Payutto), Phochananukrom Phutthasasana, (Dictionnaire du bouddhisme), chabap pramoan sap, Bangkok, 2538 (1995), p. 391-392; Bancop Bannaruci, Asiti Maha Savok, Bangkok, 2536 (1993); Phra Thep Visutthiyan (Ubon Nanthako), Pravat Anuphuttha Sangkhep 99, Bangkok, 2538 (1995). Cette seconde liste ne contient en commun avec le «khāthā » du nord de la Thaïlande qu'un peu plus d'une quarantaine de noms.

Pour un examen particulier des traditions relatives à Gavampati, l'un des quatre-vingts disciples du Buddha, on lira la contribution de F. Lagirarde au présent volume.

71. Dans ces publications, il n'est question à aucun moment de l'existence de deux listes (au moins).

72. Cf. infira note 121 .

73. Des recherches futures montreront peut-être que cette liste fut connue préalablement en Birmanie ou en pays môn.

74. C'est ce qu'indique en tout cas l'orthographe des noms. On ne peut cependant rejeter la possibilité d'une tradition originellement sanskrite qui, par la suite, aurait été « pālisée ». Certains de nos vingt thera apparaissent par exemple dans le Divyāivadāna. 
Wat Sadet, témoignage sans doute le plus ancien que nous ayons pour cette première liste, offre à ce sujet une base de départ intéressante. Pour ne s'en tenir qu'aux vingt noms dont la mention subsiste encore (nous ne pouvons être sûr, pour les soixante autres, d'une correspondance absolue avec la liste du «khāthā » du Nord), on trouvera, dans le Dictionary of Päli Proper Names, des renvois à trente textes ou collections de textes ${ }^{75}$. La majorité de ccux-ci appartiennent au Tipitaka et à ses commentaires, mais on relève également des références à des ouvrages indépendants tel le Mahāvamsa ou le Dipavamsa. On constatera que certains de nos thera apparaissent fréquemment dans la littérature bouddhique tel Upāli qui est répertorié dans 14 textes ou collections de textes. D'autres sont beaucoup moins connus comme Pabhankara qui n'apparaît que dans l'Apadāna. Ce sont d'ailleurs les noms les moins attestés que nous considérerons avec le plus d'attention, leur rareté permettant en effet d'identifier avec beaucoup plus de précision les sources d'inspiration éventuelles des compilateurs locaux. En même temps, lorsqu'un même texte sert de référence à plusieurs noms, nous considérerons qu'il y a de fortes chances pour que ce texte ait été connu par ces compilateurs. Il en est ainsi pour les Theragàthā et leur commentaire, cités pour 13 noms $^{76}$; l'Apadāna, cité pour 11 noms $^{77}$; la collection de l'Añguttaranikàya et son commentaire, cités pour 9 noms; ainsi que la Dhammapadatthakathā citée pour 8 noms. En croisant les deux critères de classification, c'est-à-dire lorsque nous recherchons à la fois les noms qui ne sont contenus que dans un seul texte et les textes qui sont cités le plus grand nombre de fois pour l'ensemble des noms, nous obtenons une fourchette encore plus précise des références que nous recherchons. Les textes qui arrivent alors en tête sont l'Apadāna et les Theragāthă. On sait que ceux-ci appartiennent à une même collection, le Khuddakanikāya, qui comporte par ailleurs les Jãtaka, le Buddhavamsa, le Suttanipāta et l'Udāna - tous quatre relevés également comme références littéraires. Il semble donc bien que ce soient les traditions véhiculées par une certaine partie du Suttapitaka qui aient inspiré en grande partie le rédacteur de notre liste. Elles ne furent cependant pas les seules : si l'on considère la liste complète du « khāthā » du Nord (nous admettrons qu'elle est en étroit rapport avec la liste de

75. Figureront parmi les collections de textes le Vinavapitaka complet ainsi que chacun des quatre premiers nikāya du Suttapitaka qui forment des groupes homogènes. Les ouvrages du Khuddakanikāya, dans la mesure où ils ont des contenus et des formes très divers, seront traités séparément. Nous donnons ici la liste avec les abréviations de G. P. Malalasekera, indiquant celles du Critical Pāli Dictionary (qui font maintenant autorité) entre parenthèses: A (AN), AA (Mp), Ap, Bu (Bv), BuA (Bv-a), D (DN), DA (Sv), DhA (Dhp-a), DhSA (As), Dpv (Dīp), Dvy, J (Ja), KhpA (Khp), M (MN), MA (Ps), Mbv (Mhbv), Mhv, MT (Mhv-t), S (SN), SA (Spk), SN (Sn), SNA (Sn-a), Sp, Thag (Th), ThagA (Th-a), Ud, UdA (Uda), VibhA (Vibh-a), Vin, Vsm (Vism).

76. Les Theragāthā et leur commentaire - qui contiennent une liste de 264 noms de thera constituaient apparemment des sources privilégiées pour y puiser les noms des quatre-vingts disciples. En fait, elles ne présentent en commun avec la liste complète du « khāthā » du Nord que 48 noms. Cf. Phra Sut Lè Atthakhatha Plè: Khuthakanikay Therakhatha, vol. 50, 51, 52, 53 (traduction thaïe des Theragāthā), Bangkok, 2525 (1982); Suttantapitake Khuddakanikāye: Vimānavatthu, Petavatthu, Theragāthā, Therīgāthāpāli (contient l'édition thaïe des Theragāthā pāli), Bangkok, 2500 (1957). Les listes de ces deux ouvrages montrent quelques variations dans la notation des noms.

77. L'Apadāna contient les noms de 543 disciples. Il ne constitue pourtant pas la source unique de la liste qui nous occupe. Cf. Phra Sut Lè Atthakhatha Plè : Khutthakanikay Apathan, vol. 70, 71, 72 (traduction thaïe de l'Apadāna), Bangkok, 2525 (1982); Suttantapitake Khuddakanikāye : Apadānapāli (édition thaïe de l'Apadāna pāli), 2 vol., Bangkok, 2500 (1957). 
notre buddhapäda), plusieurs références échappent encore à toute identification, même en admettant la possibilité de corruptions orthographiques ${ }^{78}$.

Les huit noms de personnages inscrits dans le bandeau vertical sont ordonnés selon une logique qui est beaucoup moins évidente que précédemment. Nous trouvons dans l'ordre: Phra Mahā Kondaññathera (Aññā Kondañña Thera), Phra Kumārakassapa (Thera), Phra Kassapathera, Gayānandī (fusion de Gayākassapa et de Nadīkassapa ${ }^{79}$ ), Uruveḷakassapa, Virunahakarāja (Virūḷhakarājā), Dhaț̣haraț̣harāja (Dhataratṭharājā) et Phra Khattagāma (?). Les cinq premiers (six en réalité) sont à l'évidence liés à la liste précédente. Dans le « khāthā » du nord de la Thaïlande, ils sont en effet nommés parmi les huit thera qui précèdent Ratthapāla, le dernier de notre liste de vingt thera. Étant donné la disposition des représentations, il est évident que l'observateur du buddhapāda était invité à commencer sa lecture par la partic supérieure du côté droit (représentation de Kondañña). Il devait alors descendre jusqu'à l'angle inférieur (représentation de Khattagāma), poursuivre horizontalement vers la gauche (où se trouvent nos vingt thera) jusqu'au troisième angle (disparu) qu'il remontait sans doute jusqu'au dernier angle (également disparu). Les sixième et septième noms désignent deux des quatre grands rois (cattāro mahārajāno) qui gardent les points cardinaux ${ }^{80}$. Il s'agit en l'occurrence des gardiens du Sud et de l'Est. Ceux du Nord et de l'Ouest devaient figurer logiquement dans la partie gauche de l'empreinte aujourd'hui manquante. Le huitième personnage, Phra Khattagāma, n'a pu être identifié.

La ligne inscrite de la bande horizontale supérieure donne les noms des dix premiers des vingt-quatre Buddha antérieurs à Gotama. Il manque donc manifestement quatorze noms : ceux-ci étaient très probablement gravés à la suite, mais ont disparu. Avant le nom de Dīpankara - reconnu pour être le premier des vingt-quatre Buddha - figurent cependant encore trois autres noms, ceux des Buddha Saranankara, Medhankara et Tanhankara qui, selon le Buddhavamsa, sont antérieurs à Dïpankara bien que vivant dans le même kappa que ce dernier. Il semble donc bien que cc soit le Buddhavamsa ${ }^{81}$ - ou peut-être son commentaire ${ }^{82}$ ou un texte postérieur qui lui est apparenté ${ }^{83}$ - qui ait ici inspiré le rédacteur de notre inscription. L'ordre des Buddha est parfaitement respecté, partant du plus récent (si l'on admet que les quatorze premiers aient également été cités) jusqu'au plus ancien. L'observateur, après avoir lu les noms des quatre-vingts disciples et des quatre gardiens, terminait donc son tour de l'empreinte par la lecture des noms des vingt-quatre Buddha.

La troisième ligne inscrite horizontale est une épigraphe du donateur du buddhapāda, dont le nom est Dharmarucì. Celui-ci appelle sur lui, en raison de son acte méritoire, la fortune et la puissance. Il pouvait donc s'agir d'un grand seigneur. De l'or (150000

78. C'est le cas pour les noms suivants: Pārațtha, Pāvalī, Sodatta, Leva, Yaggika, Veyyakappa, Rohanāma, Sañghāmajitā, Pannamūlina, Supāka, Picasā, Upahạ̣na, Anāgāra et Jasira.

79. Gayā et Nadì sont deux des trois frères Kassapa, le troisième - Uruvela - étant cité ensuite dans l'inscription. Il ne fait pas de doute que le rédacteur a confondu ici les deux noms pour n'en faire qu'un seul.

80. Il est question de ces quatre grands rois dans le Dïghanikāya, l'A inguttaranikāya, la Dhammapadațhakathā, etc.

81. Les noms des 24 buddha sont également contenus dans l'introduction des Jātaka (Nidānakathā) qui est inspirée de vers provenant du Buddhavamsa (O. v. Hinüber, p. 55). P. Skilling nous informe oralement que les vingt-quatre noms figurent encore dans l'Apadāna-ațhakathā.

82. Il s'agit de la Madhuratthavilāsin̄. Le plus ancien manuscrit de ce texte a été trouvé en Thaïlande (Wat Lai Hin, au sud de Chiang Mai) et date de 1551 (Cf. O. v. Hinüber, op. cit., p. 145). Il est également cité parmi les sources pāli de la Traibhīmikathā, ouvrage siamois daté du milieu du XIV siècle (Cf. G. Coedès et C. Archaimbault, Les Trois Mondes, p. 3).

83. On citera par exemple le Sotathakimahānidāna, texte apocryphe de Thaïlande, qui fait également référence à Taṇhañkara, Medhaṅkara et Saraṇañkara. Cf. O. v. Hinüber, op. cit., p. 199. 
unités ?) et de l'argent - pour une valeur respective de 10 et de 36 «tamleung » - furent accordés à l'artisan qui réalisa l'empreinte.

\section{Le buddhapāda du Wat Chomphuvek}

Le troisième buddhapāda inscrit est celui du Wat Chomphuvek de Nonthaburi ${ }^{84}$. Il possède six textes de longueur variable, tous en pāli (écriture khmère), placés à différents endroits sur la surface de la pierre. Quatre d'entre eux sont répartis sur le grand cakra représenté au centre de l'empreinte. Le premier comporte douze lignes qui correspondent à autant de rayons du cakra. Le second est inscrit sur le moyeu. Le troisième figure dans un cercle qui entoure le moyeu. Le quatrième occupe la jante du cakra. Le cinquième est gravé sur la partie supérieure de l'empreinte, juste au-dessus des orteils. Le sixième, enfin, se présente comme une longue ligne transversale sur le côté droit.

Les six textes sont de forme et de nature différentes. Nous trouverons utile, ici, de les séparer en trois séries - la première associant le texte I au texte $\mathrm{V}$, la seconde groupant les textes II, III et IV, la troisième mettant à part le texte VI. Nous donnerons pour chacun une translittération, ainsi qu'une traduction lorsque celle-ci est possible. Nous procéderons ensuite à un commentaire.

\section{Texte I ${ }^{85}$}

*1. [dukkhāriya] saccaṇ evan tathañānam vande sirasāham

Je salue de la tête la connaissance de la vérité, c'est-à-dire la noble vérité sur la douleur.

2. (du)kkhāriyasaccam ñeyyạ̣ iti ki(ccañā)nam paname ham

Je $m$ 'incline devant la connaissance de la tâche : «la noble vérité sur la douleur doit être comprise ${ }^{86}$.

3. dukkhāriya[saccam] ñātan ti sadāham va(nde) katañānam

Je salue à chaque instant la connaissance de l'accomplissement : «la noble vérité sur la douleur est comprise $»$.

*4. dukkhodayarā (ga)tanhāriyasaccam (va)nde tathañānam

Je salue la connaissance de la vérité, la noble vérité du désir et de la passion

(comme) origines de la douleur.

5. (duk)kho[dayasac] cam hañam iti kiccañānam paname ham

Je m'incline devant la connaissance de la tâche : «la vérité sur l'origine

de la douleur doit être abandonnée ».

6. dukkhodayasaccam sammā pahinam me vande katañānam

Je salue la connaissance de l'accomplissement : « la vérité sur l'origine de la douleur est par moi parfaitement abandonnée ».

84. Charïk Nai Prathet Thai, lem 5, p. 113-119; Prajum Silacharïk Phak Thi 4, Bangkok, 2513 E.B. (1970), p. 6-10. On trouvera une approche légèrement différente des inscriptions dans Suphaphan na Bangchang, Wiwathanakan Ngan Khien Phasa Bali Nai Prathet Thai : Chariik Tamnan Phongsawadan San Prakat, Bangkok, 2529 E.B. (1986), p. 29-33.

85. Les portions restituées par les éditeurs figurent entre parenthèses, nos propres conjectures figurant entre crochets. Le texte est apparemment versifié, chaque ligne étant composée de trois pāda de six syllabes. Dans la mesure où le nombre de ces dernières est de 216 , soit $6 \times 4 \times 9$, il faut peut-être considérer que nous avons ici des strophes de type tamımajjhă. Le rythme est respecté, à quelques exceptions près.

86. Le terme « iti » introduit un discours direct. Ce sont les paroles que le Buddha proféra dans le Parc aux gazelles qui sont ici répétées. Cf. Vin. I, Mahāvagga, p. 10-12; A. Bareau, Recherches sur la biographie du Buddha dans les Sütrapitaka et les Vinayapitaka anciens, (PEFEO, 53), 1963, p. $172-173$. 
*7. dukkhūpasamo yam mokkhāriyasaccam vande tathañānam

Je salue la connaissance de la vérité, la noble vérité sur la libération qui est apaisement de la douleur.

8. dukkhūpasamo me ti ... kiccañanam paname ham

Je m'incline devant la connaissance de la tâche : «l'apaisement de la douleur ... ».

9. dukkhakkhayasaccam me sacchikatan tī (vande) katañānam

Je salue la connaissance de l'accomplissement : «la vérité sur l'extinction

de la douleur est vue de mes propres yeux ».

*10. dukkhakkhayagāmìmaggāriyasaccam (vande) tathañānam

Je salue la connaissance de la vérité, la noble vérité sur la voie menant à l'extinction de la douleur.

11. dukkhakkhayamaggo bhā ... [kic]cañānam paname ham

Je m'incline devant la connaissance de la tâche : « la voie de l'extinction de la douleur (doit être cultivée) ».

12. dukkhakkhayagāmì me bhāvitamaggo vande katañānam

Je salue la connaissance de l'accomplissement : « la voie menant à l'extinction

de la douleur est par moi cultivée ».

Texte $\mathrm{V}^{87}$

dukkhadukkhodayam yo amatampi nirodhabāsam (-vāsam? ?) saccañānam

ñeyyam heyyāpi kiccam matam sa.ma.du. dukam bhāvitạn (kicca)ñānam

ñätankena(p)pahānam adhigatam asamo bhāvitam kattañānam

(ducch)ākāran tivațtam na siya dasabalo sețthadhammam namāmi

Celui qui a la connaissance de la vérité, c'est-à-dire la douleur, l'origine

de la douleur, la condition d'extinction et même l'immortalité -

(Celui qui a) la connaissance de la tâche, ce qui doit être compris, ce qui doit être abandonné, ce qui doit être fait (?), ce qui est pensé (?), la douleur, son origine, le chemin (?) ${ }^{88}$, ce qui est développé -

(Celui qui a) la connaissance de l'accomplissement, la compréhension, l'abandon, la réalisation, le développement, celui-là est incomparable, il est muni des dix pouvoirs ... (les douze ?) aspects et les trois cycles ... Je m'incline devant l'excellent Dharma.

Les textes I et V ont pour sujet les Quatre Nobles Vérités (catur-āriyasacca) qui furent prêchées par le Buddha lors de son premier sermon à proximité de Bénarès. Ils se rapportent plus précisément aux trois cycles (ti-parivatta) ${ }^{89}$ attachés à chacune de ces Vérités et donnant lieu aux douze aspects ou modalités (dvādasa-ākāra). Si nos deux

87. La structure, la grammaire et l'orthographe (ex : kattañānam) du texte étant incorrectes, il est impossible d'arriver à une traduction qui soit satisfaisante. Nous proposons la nôtre avec la plus grande réserve. Il s'agit apparemment d'une strophe de type saddharā $(21 \times 4)$, même si le rythme pose quelques problèmes, notamment à la ligne 1 où il manque par ailleurs une syllabe. Certaines erreurs paraissent inputables à l'auteur, d'autres relèvent peut-être d'une mauvaise lecture de l'éditeur.

88. "Sa.ma.du. » sont manifestement les initiales de samudaya, magga et dukkha.

89. Les trois cycles sont la connaissance ( $\tilde{n} \bar{n} n a)$ de la vérité (sacca ou tatha), de la tâche (kicca) et de l'accomplissement (kata) de cette tâche. Pour les deux dernières notions, Walpola Rahula (L'enseignement du Buddha, Paris, 1961, p. 124) parle respectivement de la connaissance du fonctionnement de chacune des Quatre Nobles Vérités et de la connaissance que la fonction de chacune des Quatre Nobles Vérités a été accomplie. 
textes font référence à des données issues du Tipitaka ${ }^{90}$, il est cependant manifeste qu'ils n'ont pas été tirés des sources canoniques elles-mêmes. Étant donné les incorrections de la langue et les incohérences de la formulation (en particulier pour le texte $\mathrm{V}^{91}$ ), il semble bien qu'on ait affaire ici à des compositions tardives et locales, peut-être inspirées par un (ou des) commentaire(s) que nous n'avons pu identifier.

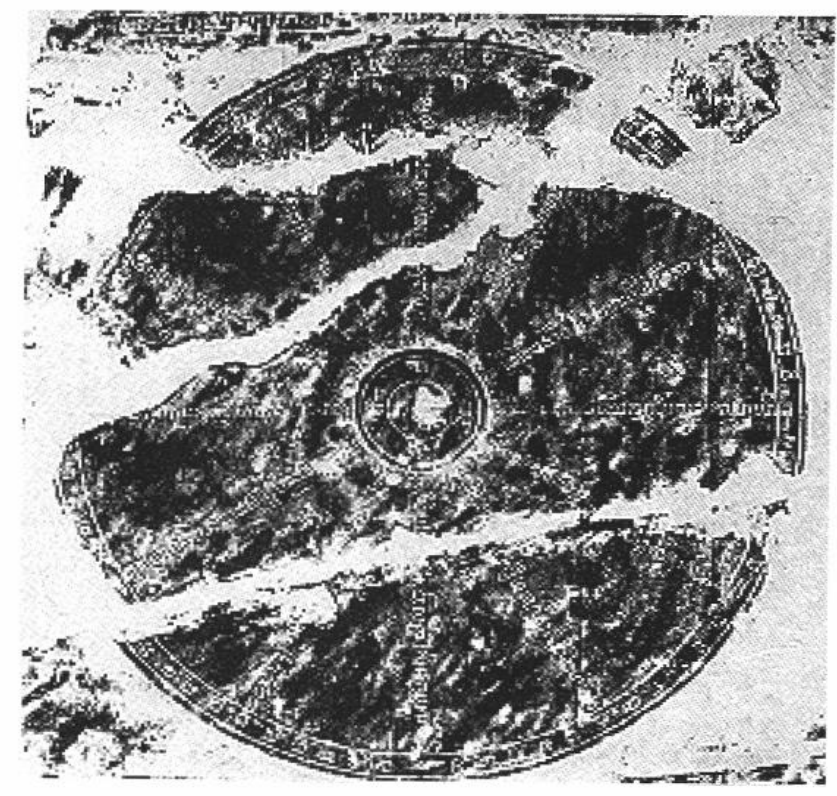

Cakra du buddhapāda du Wat Chompuvek (Nonthaburi) (Fine Arts Department)

De toutes les marques attachées à une empreinte du Buddha, la roue (skt. cakra, P. cakka) est la plus ancienne et on la trouve représentée à toutes les époques et dans tous les pays touchés par le bouddhisme. Or, il est intéressant de constater à quel point sa figuration présente des variantes selon que le contexte diffère. L'élément le plus significatif paraît être à ce sujet les rayons. Dans les sources les plus anciennes - celles du canon - nous avons déjà signalé que la roue était décrite comme présentant des rais au nombre de mille. Ce détail ne fut apparemment jamais pris à la lettre par les sculpteurs et les graveurs qui trouvaient plus commode de procéder à des divisions de $8,12,16,24$ ou 32 rayons. Si l'on observe la plupart des buddhapāda siamois ou birmans, choisis parmi les plus anciens, on peut même se rendre compte que les rayons ne possèdent en fait qu'une importance toute relative, la roue pouvant en effet être traitée en fleur de lotus (les motifs sont alors très variés), à moins qu'elle ne serve tout simplement à contenir les 108 signes auspicieux. Dans le cas précis du buddhapāda du Wat Chomphuvek, il est cependant important de constater que le nombre des rayons, fixé à douze, a un sens tout particulier. En étant associé aux douze aspects des Quatre Nobles Vérités, il donne en effet au cakra (devenu ailleurs un élément décoratif, même si sa figuration reste obligatoire) son

90. Mahāvagga du Vinaya-pitaka (éd. H. Oldenberg, I, p. 11) et Dhammacakkappavattanasutta du Samyuttanikāya (éd. L. Feer, II, p. 422).

91. On peut établir une grille de correspondances entre les trois premières lignes, chacune faisant référence aux Quatre Nobles Vérités inscrites à l'intérieur d'un cycle. La synthèse est faite dans la quatrième ligne, avec mention implicite du Buddha (yo, asamo, dasabalo) et intervention du rédacteur. Certains termes dérangent cependant le schéma d'ensemble. 
véritable caractère de symbole sacré. Par lui (et par le texte qui constitue son support), c'est en effet l'épisode où le Buddha met en mouvement la roue de la Loi qui se trouve explicitement rappelé. La représentation même de l'empreinte pourrait ainsi pratiquement passer au second plan, n'ayant pas d'autre objet que celui de servir de support au dharmacakra, plus vénéré que le buddhapāda lui-même. Cette remarque s'avère importante dès lors que l'on essaie de procéder à une datation. En s'intéressant davantage à la roue, dont la représentation monumentale fut particulièrement en faveur dans la plaine de la Mè Nam Chao Phraya entre le $\mathrm{VI}^{\mathrm{c}}$ et le VIII ${ }^{\mathrm{c}}$ siècle ${ }^{92}$ - alors qu'on en trouve plus d'exemples dans l'histoire de l'art siamois -, il est en effet possible d'assigner à notre objet une date antérieure à celle qui lui est habituellement donnée, en l'occurrence le $\mathrm{XX}^{\mathrm{c}}$ siècle de l'ère bouddhique (mil. XIV $\left.\mathrm{V}^{\mathrm{e}}-\mathrm{mil} . \mathrm{XV}^{\mathrm{e}}\right)^{93}$. On mettra d'autant plus en évidence le parallèle entre notre buddhapāda et les dharmacakra monumentaux anciens, que certains de ces derniers possèdent justement des inscriptions faisant référence aux Quatre Nobles Vérités, ainsi qu'à leurs trois aspects et à leurs douze modalités ${ }^{94}$. La culture dont relève l'empreinte du Wat Chompuvek n'est donc pas forcément siamoise.

Texte II

yo bu(ddho) tam muni.

Texte III

ra lo na ha nam me ra () to () bha tve

Texte IV

1) ... da ...gha - 2) ... pilanitivo - 3) (du)kkhodayam ati - 4) (du)jjaham - 5)

(du)ssamam subha - 6) ama...ca - 7) (du)kkhanissarani - 8) (du)ttamanacagha-

9) vandetanjali - 10) (du)kkhanāyani - 11) dutțatätatha-12) ....

Le texte II est une courte formule définissant le Buddha qui n'offre aucune caractéristique particulière. Le texte III apparaît sous la forme d'un mantra dont il est difficile de préciser la signification. On remarque pourtant qu'il contient douze syllabes (dont deux illisibles) et l'on fera le parallèle avec le nombre des aspects ou modalités. Quant au texte IV, son état très lacunaire empêche une identification satisfaisante. Tout au

92. C. Bauer («Notes on Mon Epigraphy », JSS, 79/1, 1991, p. 50) donne une liste de dix dharmacakra inscrits qui ont été retrouvés dans les régions de Lopburi, Chainat et Nakorn Pathom et relèvent apparemment tous d'une culture mône. Plus récemment, R.L. Brown (The Dväravati Wheels of the Law and the Indianization of South East Asia, Studies in Asian Art and Archacology, vol. XVIII, Leiden/New York/Köln, 1996, p. 93-120) a complété cette liste avec notamment des cakra inscrits provenant de Si Thep.

93. S. na Bangchang (op. cit.) se démarque un peu de l'avis officiel (qui vise à rattacher l'empreinte aux débuts de la période d'Ayuthya) et propose une date autour de 1250 . Ses critères sont d'ordre paléographique.

94. Cf. P. Skilling, «New Pāli Inscriptions from Southeast Asia», JPTS, 23, p. 148-150; S. na Bangchang, op. cit., p. 34-37; Charïk Nai Prathet Thai, lem 1, p. 59-64, 98-99. La formulation et les caractères d'écriture sont cependant différents de ceux de l'empreinte du Wat Chompuvek. Des dharmacakra trouvés à Nakorn Pathom donnent un vers relatif aux trois cycles et aux douze aspects que l'on retrouve notamment dans la Pathamasambodhi, ouvrage bouddhique pāli composé ultérieurement, vraisemblablement en Thaïlande ( $c f$. P. Skilling, op. cit. ; et G. Cœdès, "Une vie indochinoise du Buddha ", in Mélanges d'indianisme à la mémoire de Louis Renou, 1968, p. 225-26). R. L. Brown (op. cit., p. 117-118) et H. W. Woodward (Studies in the Art of Central Siam : 950-1350, Ph.D. dissertation, Yale University, 1975, vol. I, p. 16) établissent également un parallèle entre notre buddhapāda et les cakra de l’époque Dvāravatī. 
suivrons d'une façon générale l'interprétation proposée par A. Chaem Kèo ${ }^{100}$ qui a été le seul, jusqu'à présent, à les étudier.

Texte I

\section{Namo buddhāya}

La formule est pāli et consiste en un hommage rendu au Buddha. Dans le contexte que nous étudions, elle a peut-être un sens plus complexe en rapport avec des conceptions tantriques dont la péninsule Indochinoise fournit bien d'autres exemples ${ }^{101}$. Les cinq syllabes qui la composent sont séparées, chacune d'elles étant inscrite isolément sur une phalange de l'empreinte. Elles désigneraient alors respectivement les cinq Buddha: Kukkusandha, Konāgama, Kassapa, Gotama et Ariyametteya. À un second niveau de compréhension, ces cinq syllabes correspondent également aux cinq éléments (dhātu) primordiaux, auxquels des nombres sont attachés. Sont alors sous-entendus l'eau $(\bar{a} p o d h \bar{a} t u)=12$, la terre $($ patavīdhātu $)=21$, le feu $($ tejodhātu $)=6$, le vent $($ vāyodhātu $)=$ 7 et l'éther $(\bar{a} k \bar{a} s a d h \bar{a} t u)=10$. Le total donne le nombre 56 qui est celui des qualités ou des vertus (guna) attribuées au Buddha, telles qu'elles sont contenues dans la célèbre formule «itipiso bhagavā...». La moitié du nombre des qualités du Buddha (28) additionnée au nombre affecté à l'élément éther (10) donne le nombre 38 qui est celui des qualités du Dharma, contenu dans la formule «svākkhāto bhagavatā dhammo ... ». Le quart du nombre des qualités du Buddha (14) donne quant à lui le nombre des qualités du Sangha, contenu dans la formule «supatipanno bhagavato sāvakasangho ...». Additionnées, les qualités du Triple Joyau sont donc au nombre de 108 qui est également celui des marques auspicieuses ${ }^{102}$. À notre connaissance, il n'existe aucun texte établissant explicitement un lien entre le nombre des guna et celui des mangala attachés à un buddhapāda. Dans le cas de notre empreinte, il est cependant probable que la correspondance soit pertinente.

Texte II

$$
\text { samadat }
$$

Seul le premier terme - samadat - pourrait correspondre ici à un mot, à condition d'en restituer l'orthographe exacte. Il s'agirait alors du verbe conjugué (aoriste, $3^{\mathrm{c}} \mathrm{p}$. du sg.) sanskrit sam-adāt (donna). Les autres termes sont tous composés à partir d'initiales qui ont un sens propre. Pour celles de la première combinaison - $n(a)-m(a)-b h(a)$ - $d(a)$ - elles correspondraient, comme pour l'exemple précédent, à la mention des Buddha et des éléments primordiaux. La différence est ici que seule la consonne est prise en compte, et que la dernière $-y(a)$ - ne figure pas ${ }^{103}$. L'explication donnée par Chaem Kèo sur ce

100. Silapakorn, 37ªnnée, vol. 3, p. 45-58, repris dans Nangseu Thiraleuk Phra Raja Phithi Yok Mongkhut le Somphot Phra Phutthabat Changvat Saraburi, p. 258-267. L'auteur se base principalement sur les travaux de Thep Sarikaput, Khamphi Hoa Tiai 108 et Phuttamon Osot.

101. $C f$. les travaux de F. Bizot. Pour les différentes correspondances établies avec la formule "Namo buddhāya", on consultera en particulier Le figuier à cinq branches, Recherches sur le bouddhisme khmer I, (PEFEO, 107), 1976, p. 125-128.

102. Pour la formule «itipiso ... » relative aux 108 guna, on se réfèrera à $\mathrm{F}$. Bizot \& $\mathrm{O}$. v. Hinüber, La guirlande de Joyaux, (Textes bouddhiques du Cambodge, 2), EFEO/FEM, 1994.

103. Il y a cependant un problème avec le «bh(a)» qui aurait dû être «b(a)». On remarquera par ailleurs que l'avant-dernière syllabe, qui aurait dû être « dh(a) » (si l'on considérait l'initiale de la 4 " syllabe) est devenue « $\mathrm{d}(\mathrm{a})$ » (qui est la finale de la $3^{\mathrm{c}}$ syllabe). Il semble en fait que ce soit ce dernier 
dernier point est que nous n'avons ici la référence qu'à quatre des éléments primordiaux, celui de l'éther manquant en raison de son caractère aléatoire. Pour ce qui est des Buddha, on peut arguer peut-être que comme il est fait état d'un acte accompli (samadat), la référence à Ariya Metteya (dont la venue ne s'est pas encore produite) manquerait de

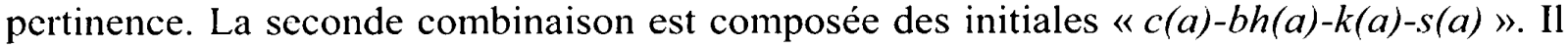
s'agirait d'un « khāthā » prononcé plus spécialement à l'intention de Sāriputta. Les lettres résumeraient alors quatre fondements du Dharma : caja dujjanasamsaggam (abandonne la fréquentation des gens mauvais!), bhaja panditasevanam (fréquente la société des sages!), kara puñnam ahorattam (produis des actes méritoires le jour comme la nuit!) et sara niccam aniccatam (souviens-toi constamment de l'impermanence !). Encore une fois, les initiales pourraient être associées aux noms des quatre premiers Buddha. La troisième combinaison « $m a-a-u$ » reproduit la fameuse formule $\langle a-u-m$ » (om) issue de la tradition brahmanique. Dans le contexte bouddhique qui nous intéresse, sa signification est cependant autre, les trois lettres désignant en effet le triple Joyau : «a» = araham (l'arahant = le Buddha); "u » = uttadharmo (le Dharma dit) et "ma " = mahäsangho (le Grand Sangha). La quatrième combinaison - «isvāsu » - se décompose en trois initiales qui sont celles des trois célèbres formules consacrées aux qualités respectives du Buddha (i-tipiso ...), du Dharma $(s v \bar{a}-k k h a t o \ldots)$ et du Sangha (supatipanno ...).

Texte III

phra araham

La mention de l'état d'arahant se réfère principalement à la personne du Buddha.

Texte IV

$$
\begin{array}{ll}
\text { itipiso visese-i } i & \text { isese buddhanāme- } i \\
\text { imena buddha tamso- } i & \text { isotam bhuddhapiti-i }
\end{array}
$$

Le texte est construit encore une fois comme un «khāthā », c'est-à-dire qu'il doit sa pertinence à un agencement de syllabes considérées comme sacrées. Il est difficile de dire exactement à quoi il est fait référence. La formule "itipiso » apparaît de façon explicite par deux fois, une première fois en introduction dans l'ordre normal, une seconde fois en conclusion où elle est écrite à rebours, la dernière syllabe " $s O$ » étant cependant coupée du reste par «tambuddha» (lui, le Buddha ?). Mis à part « $v i »$ et «bhu», les autres syllabes ( «se », «ddha», «nā», «me » et «tam») ne sont pas contenues dans le « khāthā » classique recensant les cent-huit guna, et il faut donc les chercher ailleurs. On notera que la syllabe « $\mathrm{i} »$ introduit et conclut chacun des quatre $p \bar{a} d a$ (on la retrouve donc à huit reprises), ce qui à l'évidence ne résulte pas d'un hasard. Le chiffre total des syllabes (32) peut être pertinent. Le « khāthā » « itipiso visese-i ... » demeure connu aujourd'hui, même s'il ne semble pas qu'il soit d'un usage tout à fait courant. Sa mention apparaît dans les manuels de mantra ${ }^{104}$ sous deux noms différents : "Mankuta (P. makuta) Phra Chao » ou «Mankuta Phra Phuttha Chao » = «la couronne du Maître ou du Buddha » - et "Phra itipiso Reuan Tia » (« le vénérable itipiso de la maison basse »). Comme d'autres formules

agencement - «na-mo-hu-ddhā-ya»-qui soit plutôt de règle dans l'écriture des yantra. Cf. F. Bizot, « Notes sur les yantra bouddhiques d'Indochine », in Tantric and Taoist Studies, 1981, p. 157.

104. Cf. Phra Raja Khru Vamathep Muni \& Urakhin Viriyaburana, Khamphi Khatha 108 (ou Phra Khatha 108 Phitsadan), 2516 (1973), p. 99 ; Phra Khru Sri Phromasophita (Phe Khemangkaro), Khu Mii Hvai Phra Suat Mon Lè Tamnan Phra Pathima, 2512 (1969), p. 110 ; Jotako Phikkhu, Phra Katha Akhara Maha Phutthathikhun. Bangkok, 2498 (1955), p. 66,73; Thep Sarikabut, Phutthavet Maha Mon 108, Bangkok, 2520 (1977), p. 38-39 ; Thep Sarikabut, Phutthamon Osoth, Bangkok, 2520 (1977), p. 33. 
magiques, il est utilisé essentiellement à des fins protectrices : contre les armes en période de guerre, mais également contre la maladie, les génies malfaisants et bien d'autres ennuis qui peuvent par ailleurs se présenter. Il serait prononcé également lors de certaines cérémonies telles le mariage ou la coupe du toupet des enfants. La référence qui est faite à la «maison basse » s'explique par le fait que si l'on médite sur la base de ce " khāthā ", les choses les plus élevées, et donc les moins accessibles, voient leur hauteur soudainement réduite. Un texte rapporte également la gestuelle à accomplir lorsque le « khāthāa » est récité. Nous n'avons cependant aucune information relative à des sources littéraires. Tout ce que nous pouvons conclure, c'est que l'utilisation de cette formule apparaît manifestement très ancienne. Un fait important mérite ici d'être signalé : nous avons l'exemple actuel d'un yantra thaï, en caractères khmers, constitué par quatre des formules mentionnées dans notre buddhapāda ${ }^{105}$. Le diagramme, de forme carrée, contient en effet sur son rebord la formule «itipiso visese- $i$ », et enferme trois autres carrés où se lisent dans un agencement symétrique les « khāthā » que sont «a-u-m», «isvāsu» et «namobuddhāya».

\section{Le buddhayugalapāda du Wat Pavaranivesa (Bovon Nivet)}

Nous ne saurions terminer cette revue des premiers buddhapāda siamois sans évoquer la grande dalle $(3,60 \times 2,17 \times 0,20 \mathrm{~m})$ représentant une double empreinte du Buddha (buddhayugalapāda) actuellement déposée au Wat Pavaranivesa à Bangkok ${ }^{106}$. Une inscription en pāli, gravée sur une des tranches de la pierre, commémore la fondation de l'objet sacré à une date correspondant au 11 avril 1426, durant le règne de Pal Möań ou Paramapāla (Mahādharmarājādhirāja IV : 1419-1438). Le buddhayugalapāda est par ailleurs encadré par une large bande où sont figurés quatre-vingts grands disciples du Buddha avec leur nom inscrit. La provenance de la dalle n'est pas clairement établie. Selon G. Codès, elle aurait été ramenée de Sukhothai durant la première moitié du XIX siècle ${ }^{107}$. A. B. Griswold suppose cependant qu'elle est originaire de Phitsanulok dans la mesure où il tient pour acquis que Mahādharmarāja IV résidait dans ce «muang » en $1426^{108}$. Nous ne nous attarderons pas ici sur la dimension historique de l'inscription. Il nous suffira de commenter certains détails contenus dans le texte ainsi que l'iconographie du buddhayugalapāda.

On remarquera en premier lieu la forme et le style du texte en pāli. Il s'agit en effet d'une narration - c'est-à-dire d'une composition personnelle - alors que nous ne disposions jusqu'à présent, pour les buddhapāda, que de formules stéréotypées directement inspirées par la littérature bouddhique. Le fait traduit une plus grande familiarité avec la langue sacrée, même si le texte est entaché d'erreurs ou de formes sanskritisantes ${ }^{109}$. On notera par ailleurs l'emploi d'une rhétorique que nous pourrons qualifier de conventionnelle, ainsi qu'une certaine imprécision dans la formulation - le contenu pouvant en effet donner lieu à des interprétations différentes.

105. Cf. Thep Sarikabut, Phuttharat Mahayan 108 Lè Naphisadan, Bangkok, 2518 (1975), p. 48.

106. On trouvera une présentation de cette double empreinte ainsi qu'une traduction de son inscription dans: Fournereau, Le Siam ancien, I, Paris, 1895, p. 242-254; G. Coedès, Recueil des Inscriptions du Siam, I, Bangkok, 1924, p. $151-156$ (il s'agit de l'inscription n ${ }^{\circ} 12$ ) ; A. B. Griswold \& P. ṇa Nagara, «Epigraphic and Historical Studies $N^{\circ} 20$ : The Buddhapāda of Vat Pavaranivesa and its Inscription ", JSS, 66/2, 1978, p. 112-122. Chariük Samay Sukhothai (p. 265-268) fournit une translittération et une traduction en thaï.

107. Op. cit., p. 151 .

108. Op. cit., p. 761.

109. Cf. la traduction et les notes de A. Barth dans Fournereau, op. cit., p. 249-254. 
L'inscription fait référence à deux rois - Mahādhammarāja et son fils Paramapāla Dhammarāja - mais également à trois religieux. Le premier est le mahāthera Vidyāvamsa que l'on nous présente comme le personnage qui aurait ramené de - ou à - Sukhothai la dalle où la double empreinte et l'inscription furent gravées. Le second et le troisième ont le même nom - celui de Siri Sumedhañkara - mais l'un est sangharāja et maître de l'autre qui est sanghanāyaka. Tous deux appartiennent à la communauté des vanaväsin. On peut à ce sujet opérer un parallèle avec la stèle du Wat Pa Deng antérieure de quelques années.

L'auteur du buddhayugalapāda (inscriptions comprises) est le sañghanāyaka Siri Sumedhankara. Celui-ci nous dit que l'image des deux pieds du Sugata (le "Bienvenu »= le Buddha) est conforme à l'empreinte déposée sur le Samantaküta sur l'île de Lañka. On retrouve donc là la tradition légitimante déjà invoquée par Ldaiya. On notera avec un intérêt particulier l'emploi du nom Samantakūta qui diverge de celui de Sumanakūta jusqu'ici employé dans nos inscriptions. En fait, dans le Mahāvamsa, il a déjà été relevé que les deux noms alternaient pour désigner la même entité géographique ${ }^{110}$. Ce fait contribue à renforcer notre opinion selon laquelle la célèbre chronique cinghalaise est bien la source d'inspiration utilisée par les lettrés de Sukhothai à propos des buddhapāda. Il n'est cependant pas fait mention ici d'un voyage à Larikā. S'il est fait référence aux centhuit signes auspicieux étudiés plus tôt, on remarque, à partir d'un examen de la double empreinte elle-même, que ceux-ci sont disposés selon un modèle très différent de celui que nous pouvions constater dans les empreintes de Sukhothai les plus anciennes, telles celles du Wat Traphang Thong et du Khau Nang Thong. À l'ordonnancement quadrillé et rectiligne (sauf pour le talon, traité en arcs de cercle), typique également des premiers buddhapāda de Pagan, succède en effet une représentation circulaire et totalement centrée, comme on la retrouve par exemple sur le buddhapāda du Wat Sadet. Tous les signes sont alors enfermés en fonction d'un axe radial dans le cakra qui, pour l'occasion, a un diamètre équivalent à la largeur d'une empreinte. Nous avions déjà noté le caractère artificiel de l'argumentation de L daiya selon laquelle les buddhapāda de Sukhothai étaient à l'image de l'empreinte du Sumanaküta. Les observations d'ordre stylistique nous renforcent ici dans cette opinion.

La référence faite dans l'inscription au nom de Siri Sumedhankara a donné lieu à quelques commentaires. G. Cœdès a été le premier à s'exprimer à ce sujet. Il remarque en effet qu' "il est probable qu'un des deux Sirisumedhañkara mentionnés ici est identique au Mahāmedhañkarathera nommé dans le Sāsanavamsa ${ }^{111}$ et dans la Jinakälamālin̄ī ${ }^{112}$. C'était un religieux de Jian Mai [Chiang Mai] qui s'était rendu à Ceylan en 1423 avec trente-deux de ses confrères et qui, à son retour, fonda dans diverses villes du Siam la secte des Sïhalabhikkhus. Les deux textes précités s'accordent à faire passer Mahāmedhankara par Sukhodaya $~^{113}$. La discussion de ces arguments mériterait déjà une longue étude qui n'a pas sa place ici. Qu'il nous suffise cependant de remarquer le danger qu'il y a à identifier deux personnages sur la base principale qu'ils possèdent le même nom. Celui de Medhankkara, désignant au départ un des Buddha antérieurs mais également

110. Cf. supra note 22 .

111. B. C. Law (trad.), The History of the Buddha's Religion, London, 1952, p. 56. La présentation des faits est très laconique et diffère fort de celle contenue dans la Jinakālamālinī.

112. G. Cœdès, « Documents sur l'histoire politique et religieuse du Laos Occidental », BEFEO, 25, 1926, p. 104-107 ; N. A. Jayawickrama, The Sheaf of Garlands of the Epochs of the Conqueror, London, 1968, p. 129-133.

113. Recueil des inscriptions du Siam, I, p. 152. 
appliqué à plusieurs thera cinghalais auteurs d'ouvrages en pāli ${ }^{114}$, devait être courant au $\mathrm{XV}^{\mathrm{c}}$ siècle. La meilleure preuve en est déjà que ce même nom est porté par deux des vénérables de notre inscription. A. B. Griswold et P. na Nagara, s'ils ne se prononcent pas sur l'identité du sañghanāyaka Sumedhañkara, n'hésitent pourtant pas à identifier le sangharāja Sumedhankara avec le thera Medhankara supposé être l'auteur du Lokadipakasāra (également appelé Lokappadipakasāra), texte cosmologique apparemment composé en pays môn au XIV siècle $^{115}$, voire avec le religieux de Martaban invité par Ldaiya en $1361^{116}$. Les arguments qui nous sont donnés apparaissent séduisants mais ne constituent sans doute pas une preuve suffisante pour l'identification. Une étude approfondie du Lokadipakasāra serait cependant à souhaiter. Sa relation avec la Traibhīmikathā, autre ouvrage cosmologique présumé du XIV ${ }^{e}$ siècle, reste à préciser ${ }^{117}$.

À la fin du XIX ${ }^{e}$ siècle, déjà, l'état de détérioration de la pierre empêchait Fournereau de lire la plus grande partie des quatre-vingts noms de disciples gravés autour des deux empreintes. Afin de restituer l'ensemble de la liste, il s'appuya alors sur des inscriptions sur marbre conservées au Wat Suthat de Bangkok ${ }^{118}$. Elles étaient gravées sur les statues de quatre-vingts sāvaka entourant le Buddha, et il semblait acquis, à l'époque, qu'elles reproduisaient chacun des noms figurant sur le buddhayugalapāda. Le résultat est cependant loin d'être satisfaisant. Fournereau avait en effet recopié des inscriptions qu'il ne pouvait lire et sa copie avait été ensuite transcrite par le R. P. Schmitt, érudit local auquel on faisait appel pour traduire un certain nombre de textes en langues khmère ou thaï ${ }^{119}$. A. Barth, le savant bien connu, fut mis ensuite à contribution afin d'interpréter la liste obtenue. Il reconnut qu'il s'agissait selon toutes probabilités de la liste des quatrevingts disciples principaux du Buddha, mais il tempéra son jugement en précisant qu'il ne se souvenait pas en avoir déjà rencontré une énumération complète. Un certain nombre de noms lui parurent par ailleurs étranges. Il releva également les incorrections du pāli et les mélanges avec l'orthographe sanskrite. Il conclut enfin que plusieurs des noms étaient impossibles et entièrement corrompus ${ }^{120}$. Il est en effet difficile, lorsque l'on se reporte à la liste de Fournereau, d'identifier une bonne moitié des références. Les autres, reconnaissables malgré une orthographe souvent fautive, sont toutes contenues dans la liste qui nous est fournie par la Paramatthadipanī ${ }^{121}$ et qui constitue aujourd'hui la seule liste véritablement reconnue en Thaïlande. Les lettrés thaïs contemporains semblent d'ailleurs avoir établi l'équivalence dans la mesure où un ouvrage récent consacré aux

114. Dictionary of Pāli Proper Names, vol. II, p. 663.

115. À propos du Loka(ppa)dipakasāra composée par Medhañkara : O. v. Hinüber, A Handbook of Päli Literature, p. 183-184 ; N. Ray, Theravāda Buddhism in Burma, p. 175 ; B. C. Law, op. cit., p. 47 et 53 ; M. H. Bode, The Pāli Literature of Burma, p. 35-36. Il existe une édition siamoise du texte avec traduction en thaï (National Library, Bangkok, 1986). Cf: le compte rendu de P. Skilling dans Buddhist Studies Review, 7 (1990), p. 120.

116. Op. cit., p. 119.

117. Il existe une hypothèse selon laquelle le Lokadipakasāra pourrait être l'équivalent pāli de la Traibhümikathā (cf. introduction de l'édition siamoise de Loka-d. - signalée par O. v. Hinüber, op. cit., p. $184, \S 397)$. Ce point de vue est contesté par P. Skilling (communication personnelle).

118. Le Siam ancien, I, op. cit., p. 62-64 et 245-247.

119. Notamment des annales et des inscriptions publiées par la Mission Pavie.

120. Le Siam ancien, I, op. cit., p. 248.

121. La Paramatthadīpan̄i, cuvre de Dhammapāla, forme le commentaire de six textes du Khuddhakanikāya: l'Udāna, l'Itivuttaka, le Vimānavatthu, le Petavatthu, les Theragāthāa, les Therīgāthā et le Cariyāpitaka. Les Theragāthā donnaient une liste de 264 thera. Leur commentaire spécifique isole parmi ceux-ci une liste de 80 mahāsāvakā (cf. O. von Hinüber, op. cit., p. 141 ; Bancop Bannaruci, Asiti Maha Savok, Bangkok, 2536 (1993), p. 2). 
disciples du Buddha et contenant la liste orthodoxe des mahã asīti sāvakā fut justement imprimé au Wat Bovon Nivet où se trouve notre buddhayugalapäda ${ }^{122}$. Il est pourtant loin d'être évident que le commentaire de Dhammapāla fut la référence littéraire utilisée par le graveur des quatre-vingts plaques et de notre empreinte. Il paraît en effet impossible d'établir une véritable correspondance pour l'autre moitié des noms contenus dans la liste de Fournereau. Cette dernière est par ailleurs très différente de la liste apparemment commune à l'empreinte du Wat Sadet et au «khāthā » du nord de la Thaïlande. Plusieurs noms, en effet, aisément identifiables dans l'une, ne se retrouvent pas dans l'autre. Il est donc tentant, dans l'état actuel des connaissances, de suggérer l'existence d'une troisième liste (aujourd'hui oubliée ?) de quatre-vingts disciples, variante de celle qui nous est fournie par la Paramatthadipanī.

Un tour d'horizon des buddhapāda siamois les plus anciens nous a permis de mettre en évidence un certain nombre de caractères originaux. Contrairement aux empreintes plus tardives, que les annales locales mettent en rapport avec une venue du Buddha en pays t'ai ( siamois, yuan, lao, etc.), celles du XIV et du XV $\mathrm{XV}^{\mathrm{e}}$ siècles, liées à la culture de Sukhothai, insistent sur une prétendue filiation avec un modèle situé à Ceylan. Nous avons vu que ce lien était probablement factice - ne serait-ce que pour des raisons iconographiques - et qu'il procédait manifestement de références littéraires. La source principale fut probablement le Mahāvamsa, dont on peut supposer qu'il fut connu très tôt à Sukhothai. L'influence de la Birmanie dut alors jouer un rôle déterminant, soit par un contact direct avec Pagan, soit par des relais qui furent peut-être môns. C'est à partir des côtes de la mer d'Andaman, en effet, que se diffusèrent dès la fin du XII ${ }^{c}$ siècle des traditions bouddhiques régénérées à Ceylan et sans doute fort distinctes de celle que connut la civilisation antérieure de Dvāravatì. Parmi les vestiges qui se rattachent d'une façon ultime à cette dernière, il nous faudra peut-être ajouter un buddhapāda qui, jusqu'à présent, était associé à la civilisation d'Ayuthya. À Sukhothai, les premières empreintes du Buddha sont contemporaines des premières inscriptions. Les unes et les autres nous permettent, d'une façon complémentaire, de mieux appréhender la culture bouddhique siamoise à ses origines. Elles nous apportent sur les connaissances religieuses de l'époque de précieux indices et éclairent du même coup certains fondements d'une nouvelle idéologie politique (modèle du souverain cakravartin). Les références littéraires apparaissent alors nombreuses. On note parmi les plus anciennes des textes cinghalais relatifs à la tradition des cent-huit marques auspicieuses. Ces textes, il est utile de le remarquer, étaient largement postérieurs aux œuvres canoniques et post-canoniques, et contribuèrent sans doute à donner au bouddhisme sa nouvelle vigueur. En Asie du Sud-Est, ils sont donc étroitement liés à la vague de cinghalisation. À partir de la seconde moitié du XIVe siècle, et cela probablement jusqu'au $\mathrm{XVI}^{\mathrm{c}}$, on voit se développer sur les buddhapäda une iconographie nouvelle fondée sur d'autres sources littéraires. Le canon et ses commentaires paraissent être souvent une source directe d'inspiration. Certaines références, cependant, se laissent difficilement identifier et relèvent apparemment de traditions originales et régionales, parfois totalement étrangères à l'orthodoxie cinghalaise. Le buddhapāda, dans ce cas, peut devenir le support de conceptions tantriques et se rattacher à un fonds de pratiques reconnues aujourd'hui comme très anciennes. Il est intéressant de constater que sous une forme différente - et malgré les nombreuses réformes qu'a connues le bouddhisme siamois - ces traditions marginales ont survécu. 